\title{
Control and Stabilization of the Korteweg-de Vries Equation on a Periodic Domain
}

\author{
Camille Laurent \\ Laboratoire de Mathématiques \\ Université Paris-Sud \\ Bâtiment 425, F-91405 Orsay Cedex, France \\ email: camille.laurent@math.u-psud.fr \\ Lionel Rosier \\ Institut Élie Cartan \\ UMR 7502 UHP/CNRS/INRIA, B.P. 239 \\ F-54506 Vandœuvre-lès-Nancy Cedex, France \\ email: rosier@iecn.u-nancy.fr \\ and \\ Bing-Yu Zhang \\ Department of Mathematical Sciences \\ University of Cincinnati \\ Cincinnati, Ohio 45221, USA \\ email: bzhang@math.uc.edu
}

\begin{abstract}
This paper aims at completing an earlier work of Russell and Zhang 38] to study internal control problems for the distributed parameter system described by the Korteweg-de Vries equation on a periodic domain $\mathbb{T}$. In [38, Russell and Zhang showed that the system is locally exactly controllable and locally exponentially stabilizable when the control acts on an arbitrary nonempty subdomain of $\mathbb{T}$. In this paper, we show that the system is in fact globally exactly controllable and globally exponentially stabilizable. The global exponential stabilizability corresponding to a natural feedback law is first established with the aid of certain properties of propagation of compactness and propagation of regularity in Bourgain spaces for solutions of the associated linear system. Then, using a different feedback law, the resulting closed-loop system is shown to be locally exponentially stable with an arbitrarily large decay rate. A time-varying feedback law is further designed to ensure a global exponential stability with an arbitrary large decay rate.
\end{abstract}

\section{Introduction}

The well-known Korteweg-de Vries (KdV) equation can be written as

$$
\partial_{t} u+u \partial_{x} u+\partial_{x}^{3} u=0,
$$

where $u=u(x, t)$ denotes a real-valued function of two real variables $x$ and $t$. The equation was first derived by Korteweg and de Vries [19] in 1895 (or by Boussinesq [4] in 1876 1) as a model for

\footnotetext{
${ }^{1}$ The interested readers are refereed to a nice article of de Jager 14 for the origin of the KdV equation.
} 
propagation of some surface water waves along a channel. The KdV equation has been intensively studied from various aspects of both mathematics and physics since the 1960s when solitons were discovered through solving the KdV equation, and the inverse scattering method, a so-called nonlinear Fourier transform, was invented to seek solitons [12, 25]. It turns out that the equation is not only a good model for some water waves but also a very useful approximation model in nonlinear studies whenever one wishes to include and balance a weak nonlinearity and weak dispersive effects [25]. In particular, the equation is now commonly accepted as a mathematical model for the unidirectional propagation of small-amplitude long waves in nonlinear dispersive systems.

In this paper, we consider the $\mathrm{KdV}$ equation posed on the periodic domain $\mathbb{T}$ :

$$
\partial_{t} u+u \partial_{x} u+\partial_{x}^{3} u=0, \quad x \in \mathbb{T}, t \in \mathbb{R} .
$$

The equation is known to possess an infinite set of conserved integral quantities of which the first two are

$$
I_{1}(t)=\int_{\mathbb{T}} u(x, t) d x
$$

and

$$
I_{2}(t)=\int_{\mathbb{T}} u^{2}(x, t) d x .
$$

From the historical origins [19, 4, 25] of the KdV equation, involving the behavior of water waves in a shallow channel, it is natural to think of $I_{1}$ and $I_{2}$ as expressing conservation of volume (or mass) and energy, respectively. The Cauchy problem for the equation (1.2) has been intensively studied for many years (see [39, 16, 3, 18] and the references therein). The best known result so far [15] is that the Cauchy problem is well-posed in the space $H^{s}(\mathbb{T})$ for any $s \geq-1$ :

Let $s \geq-1$ and $T>0$ be given. For any $u_{0} \in H^{s}(\mathbb{T})$, the equation (1.2) admits a unique solution $u \in C\left([0, T] ; H^{s}(\mathbb{T})\right)$ satisfying

$$
u(x, 0)=u_{0}(x) .
$$

Moreover, the corresponding solution map $\left(u_{0} \rightarrow u\right)$ is continuous from the space $H^{s}(\mathbb{T})$ to the space $C\left([0, T] ; H^{s}(\mathbb{T})\right) .2$

In this paper we will study the equation (1.2) from a control point of view with a forcing term $f=f(x, t)$ added to the equation as a control input:

$$
\partial_{t} u+u \partial_{x} u+\partial_{x}^{3} u=f, \quad x \in \mathbb{T}, t \in \mathbb{R},
$$

where $f$ is assumed to be supported in a given open set $\omega \subset \mathbb{T}$. The following exact control problem and stabilization problem are fundamental in control theory.

Exact control problem: Given an initial state $u_{0}$ and a terminal state $u_{1}$ in a certain space, can one find an appropriate control input $f$ so that the equation (1.3) admits a solution $u$ which satisfies $u(., 0)=u_{0}$ and $u(., T)=u_{1}$ ?

Stabilization problem: Can one find a feedback control law: $f=K u$ so that the resulting closedloop system

$$
\partial_{t} u+u \partial_{x} u+\partial_{x}^{3} u=K u, \quad x \in \mathbb{T}, t \in \mathbb{R}^{+}
$$

is asymptotically stable as $t \rightarrow+\infty$ ?

\footnotetext{
${ }^{2}$ If $s>-\frac{1}{2}$, this solution map is, in fact, analytic.
} 
The problems were first studied by Russell and Zhang for the KdV equation [37, 38. In their work, in order to keep the mass $I_{1}(t)$ conserved, the control input $f(x, t)$ is chosen to be of the form

$$
f(x, t)=[G h](x, t):=g(x)\left(h(x, t)-\int_{\mathbb{T}} g(y) h(y, t) d y\right)
$$

where $h$ is considered as a new control input, and $g(x)$ is a given nonnegative smooth function such that $\{g>0\}=\omega$ and

$$
2 \pi[g]=\int_{\mathbb{T}} g(x) d x=1 .
$$

For the chosen $g$, it is easy to see that

$$
\frac{d}{d t} \int_{\mathbb{T}} u(x, t) d x=\int_{\mathbb{T}} f(x, t) d x=0 \quad \text { for any } t \in \mathbb{R}
$$

for any solution $u=u(x, t)$ of the system

$$
\partial_{t} u+u \partial_{x} u+\partial_{x}^{3} u=G h
$$

thus the mass of the system is indeed conserved.

The following results are due to Russell and Zhang [38].

Theorem A: Let $s \geq 0$ and $T>0$ be given. There exists a $\delta>0$ such that for any $u_{0}, u_{1} \in H^{s}(\mathbb{T})$ with $\left[u_{0}\right]=\left[u_{1}\right]$ satisfying

$$
\left\|u_{0}\right\|_{s} \leq \delta, \quad\left\|u_{1}\right\|_{s} \leq \delta
$$

one can find a control input $h \in L^{2}\left(0, T ; H^{s}(\mathbb{T})\right)$ such that the system (1.5) admits a solution $u \in C\left([0, T] ; H^{s}(\mathbb{T})\right)$ satisfying

$$
u(x, 0)=u_{0}(x), \quad u(x, T)=u_{1}(x) .
$$

In order to stabilize the system (1.5), Russell and Zhang employed a simple feedback control law

$$
h(x, t)=-G^{*} u(x, t) .
$$

The resulting closed-loop system

$$
\partial_{t} u+u \partial_{x} u+\partial_{x}^{3} u=-G G^{*} u, \quad x \in \mathbb{T}, t \in \mathbb{R} .
$$

is locally exponentially stable.

Theorem B: Let $s=0$ or $s \geq 1$ be given. There exist positive constants $M, \delta$ and $\gamma$ such that if $u_{0} \in H^{s}(\mathbb{T})$ satisfies

$$
\left\|u_{0}-\left[u_{0}\right]\right\|_{s} \leq \delta
$$

then the corresponding solution u of (1.7) satisfies

$$
\left\|u(\cdot, t)-\left[u_{0}\right]\right\|_{s} \leq M e^{-\gamma t}\left\|u_{0}-\left[u_{0}\right]\right\|_{s}
$$

for any $t \geq 0$. 
Thus one can always find an appropriate control input $h$ to guide the system (1.5) from a given initial state $u_{0}$ to a terminal state $u_{1}$ so long as their amplitudes are small and $\left[u_{0}\right]=\left[u_{1}\right]$. A question arises naturally.

Question 1: Can one still guide the system by choosing appropriate control input h from a given initial state $u_{0}$ to a given terminal state $u_{1}$ when $u_{0}$ or $u_{1}$ have large amplitude?

As for the closed-loop system (1.7), its small amplitude solutions decay at a uniform exponential rate to the corresponding constant state $\left[u_{0}\right]$ with respect to the norm in the space $H^{s}(\mathbb{T})$ as $t \rightarrow \infty$. One may ask naturally:

Question 2: Do the large amplitude solutions of the closed-loop system (1.7) decay exponentially as $t \rightarrow \infty$ ?

A further question is:

Question 3: For any given number $\lambda>0$, can we design a linear feedback control law such that the exponential decay rate of the resulting closed-loop system is $\lambda$ ?

One of the main results in this paper is a positive answer to Question 1 as given below.

Theorem 1.1. Let $s \geq 0, R>0$, and $\mu \in \mathbb{R}$ be given. There exists a time $T>0$ such that if $u_{0}, u_{1} \in H^{s}(\mathbb{T})$ with $\left[u_{0}\right]=\left[u_{1}\right]=\mu$ are such that

$$
\left\|u_{0}\right\|_{s} \leq R, \quad\left\|u_{1}\right\|_{s} \leq R
$$

then one can find a control input $h \in L^{2}\left(0, T ; H^{s}(\mathbb{T})\right)$ such that the system (1.5) admits a solution $u \in C\left([0, T] ; H^{s}(\mathbb{T})\right)$ satisfying

$$
u(x, 0)=u_{0}(x), \quad u(x, T)=u_{1}(x) .
$$

So the system (1.5) is globally exactly controllable.

As for Question 2, we have the following affirmative answer.

Theorem 1.2. Let $s \geq 0$ and $\mu \in \mathbb{R}$ be given. There exists a constant $\kappa>0$ such that for any $u_{0} \in H^{s}(\mathbb{T})$ with $\left[u_{0}\right]=\mu$, the corresponding solution $u$ of the system (1.7) satisfies

$$
\left\|u(\cdot, t)-\left[u_{0}\right]\right\|_{s} \leq \alpha_{s, \mu}\left(\left\|u_{0}-\left[u_{0}\right]\right\|_{0}\right) e^{-\kappa t}\left\|u_{0}-\left[u_{0}\right]\right\|_{s} \quad \text { for all } t \geq 0,
$$

where $\alpha_{s, \mu}: \mathbb{R}^{+} \rightarrow \mathbb{R}^{+}$is a nondecreasing continuous function depending on $s$ and $\mu$.

Note that Theorem 1.1 follows from Theorem 1.2 and a local control result around the state $u(x)=\mu$ (similar to Theorem A) thanks to the time reversibility of the KdV equation.

The decay rate $\kappa$ in Theorem 1.2 has an upper bound

$$
\kappa \leq \inf \left\{-\operatorname{Re} \lambda: \lambda \in \sigma_{p}\left(A_{G}\right)\right\}
$$

where $A_{G}$ is the operator defined by

$$
A_{G} v=-v^{\prime \prime \prime}-\mu v-G G^{*} v
$$

with $\mathcal{D}\left(A_{G}\right)=H^{3}(\mathbb{T})$ as domain. In order to have the decay rate $\kappa$ arbitrarily large, a different feedback control law is needed. 
Theorem 1.3. Let $\lambda>0, s \geq 0$, and $\mu \in \mathbb{R}$ be given. There exists a number $\delta>0$ and a linear bounded operator $Q_{\lambda}$ from $H^{s}(\mathbb{T})$ to $H^{s}(\mathbb{T})$ such that if one chooses the feedback control law

$$
h=-Q_{\lambda} u
$$

in system (1.5)-(1.4), then the solution $u$ of the resulting closed-loop system

$$
\partial_{t} u+u \partial_{x} u+\partial_{x}^{3} u=-G Q_{\lambda} u, \quad u(x, 0)=u_{0}(x), x \in \mathbb{T}
$$

satisfies

$$
\left\|u(\cdot, t)-\left[u_{0}\right]\right\|_{s} \leq C e^{-\lambda t}\left\|u_{0}-\left[u_{0}\right]\right\|_{s} \quad \text { for all } t \geq 0,
$$

whenever $\left\|u_{0}\right\|_{s} \leq \delta$ and $\left[u_{0}\right]=\mu, C>0$ denoting a constant independent of $u_{0}$.

Note that this is still a local stabilization result. However, the feedback laws in Theorems 1.2 and 1.3 may be combined into a time-varying feedback law (as in [9]) ensuring a global stabilization with an arbitrary large decay rate.

Theorem 1.4. Let $\lambda>0, s \geq 0$, and $\mu \in \mathbb{R}$ be given. There exists a smooth map $Q_{\lambda}$ from $H^{s}(\mathbb{T}) \times \mathbb{R}$ to $H^{s}(\mathbb{T})$ which is periodic with respect to the second variable, and such that the solution $u$ of the closed-loop system

$$
\partial_{t} u+u \partial_{x} u+\partial_{x}^{3} u=-G Q_{\lambda}(u, t), \quad u(\cdot, 0)=u_{0}
$$

satisfies

$$
\left\|u(\cdot, t)-\left[u_{0}\right]\right\|_{s} \leq \alpha_{s, \lambda, \mu}\left(\left.\left\|u_{0}-\left[u_{0}\right]\right\|\right|_{s}\right) e^{-\lambda t}\left\|u_{0}-\left[u_{0}\right]\right\|_{s} \quad \text { for all } t \geq 0,
$$

where $\alpha_{s, \lambda, \mu}: \mathbb{R}^{+} \rightarrow \mathbb{R}^{+}$is a nondecreasing continuous function depending on $s, \lambda$ and $\mu$.

The following remarks are in order.

\section{Remark 1.5.}

(i) In Theorem $A$, the control time $T$ is independent of the initial state $u_{0}$ and the terminal state $u_{1}$ and can be, in fact, chosen arbitrarily small. By contrast, in Theorem 1.1, the control time $T$ depends on the size of the initial state $u_{0}$ and the terminal state $u_{1}$ in the space $L^{2}(\mathbb{T})$. Whether the time $T$ can be chosen independent of the size of $u_{0}$ and $u_{1}$ is an interesting open question.

(ii) While the decay rates $\kappa$ in Theorem 1.2 and $\lambda$ in Theorem 1.4 are independent of $u_{0}$, the constants $\alpha_{s, \mu}\left(\left\|u_{0}-\left[u_{0}\right]\right\|_{0}\right)$ or $\alpha_{s, \lambda, \mu}\left(\left\|u_{0}-\left[u_{0}\right]\right\|_{s}\right)$ are likely not uniformly bounded; i.e., it may happen that

$$
\lim _{r \rightarrow \infty} \alpha_{s, \mu}(r)=\infty \text { or } \lim _{r \rightarrow \infty} \alpha_{s, \lambda, \mu}(r)=\infty
$$

To prove our global controllability and stabilization results described above, we will as usual consider first the associated linear open-loop system

$$
u_{t}+u_{x x x}=G h
$$

and the associated linear closed-loop system

$$
u_{t}+u_{x x x}=-G Q_{\lambda} .
$$

Without much difficulty we can show by using a standard approach in control theory of linear systems that the system (1.10) is exactly controllable in the space $H^{s}(\mathbb{T})$ and that the closed-loop 
system (1.11) is exponentially stable in the space $H^{s}(\mathbb{T})$ with an arbitrarily large decay rate $\lambda$. However, how to extend the linear results to the corresponding nonlinear systems is a challenging task. Indeed, after having published their linear results [37], Russell and Zhang had to wait for several years to extend their results to the nonlinear systems [38] until Bourgain [3] discovered a subtle smoothing property of solutions of the KdV equation posed on a periodic domain $\mathbb{T}$ when he showed surprisingly that the Cauchy problem of the KdV equation (1.2) is well-posed in the space $H^{s}(\mathbb{T})$ for $s \geq 0$. This then newly discovered smoothing property of the KdV equation has played a crucial role in the proofs of Theorem A and Theorem B in [38. By contrast, establishing the global exact controllability and stabilizability for the nonlinear system (1.7) is even more challenging. After all, the results presented in Theorem A and Theorem B are essentially linear in nature; they are more or less small perturbation of the linear results. The global results presented in Theorem 1.1, Theorem 1.2 and Theorem 1.4 are truly nonlinear and their proofs demand new tools. The needed help turns out to be certain propagation properties of compactness and regularity for the KdV equation which are inspired by those established by Laurent in [20] for the Schrödinger equation. This strategy has already been successfully applied by Dehman, Lebeau, and Zuazua [11 for the wave equation, and by Dehman, Gérard, and Lebeau [10] and Laurent [20, 21] for the Schrödinger equation.

Note that for any solution $u$ of the systems in consideration, its mean value $[u]$ is invariant. Thus it is convenient to introduce the number $\mu:=[u]=\left[u_{0}\right]$, and to set

$$
\tilde{u}=u-\mu \text {. }
$$

Then $[\tilde{u}]=0$ and $\tilde{u}$ solves

$$
\partial_{t} \tilde{u}+\tilde{u} \tilde{u}_{x}+\partial_{x}^{3} \tilde{u}+(\mu+\tilde{u}) \partial_{x} \tilde{u}=G h .
$$

if $u$ solves (1.5). Throughout the paper, $\mu$ will denote a given (real) constant, $H_{0}^{s}(\mathbb{T})=\{u \in$ $\left.H^{s}(\mathbb{T}) ;[u]=0\right\}$, and $L_{0}^{2}(\mathbb{T})=\left\{u \in L^{2}(\mathbb{T}) ;[u]=0\right\}$. We shall establish exponential stability results in $H_{0}^{s}(\mathbb{T})$ for the equation

$$
\partial_{t} u+\partial_{x}^{3} u+\mu \partial_{x} u+u \partial_{x} u=-K_{\lambda} u
$$

that will imply all the results stated above.

The paper is outlined as follows.

- In Section 2, the exact controllability and stabilizability are presented for the associated linear systems.

- In Section 3, some preliminary results in Bourgain spaces, including the propagation of compactness and the propagation of regularity for the KdV equation, are provided.

- In Section 4, the stabilization of the KdV equation by a time invariant feedback control law is studied.

- In Section 5, the stabilization of the KdV equation by a time-varying feedback control law is investigated.

Finally we end our introduction with a few comments on the boundary controllability of the KdV equation posed on a finite interval $(0, L)$ :

$$
\left\{\begin{array}{l}
u_{t}+u_{x}+u u_{x}+u_{x x x}=0, \quad x \in(0, L), t>0, \\
u(0, t)=h_{1}(t), \quad u(L, t)=h_{2}(t), \quad u_{x}(L, t)=h_{3}(t) .
\end{array}\right.
$$

The problem was first investigated by Rosier [30] and has been intensively studied in the past decade. (See [30, 47, 31, 28, 32, 8, 26, 33, 22, 13, 5, 6, 23] and the references therein.) In contrast to control problems of other equations (parabolic equation or hyperbolic equations for instance), the boundary control system (1.12) has some interesting properties. 
(i) If

$$
L \in \mathcal{N}:=\left\{2 \pi \sqrt{\frac{j^{2}+l^{2}+j l}{3}} ; j, l \in \mathbb{N}^{*}\right\}
$$

the linear system

$$
\left\{\begin{array}{l}
u_{t}+u_{x}+u_{x x x}=0, \quad x \in(0, L), t>0 \\
u(0, t)=h_{1}(t), \quad u(L, t)=h_{2}(t), \quad u_{x}(L, t)=h_{3}(t) .
\end{array}\right.
$$

associated to (1.12) is not exactly controllable if $h_{1}=h_{2} \equiv 0$. However, the nonlinear system (1.12) is locally exactly controllable (still with $\left.h_{1}=h_{2} \equiv 0\right)$ [30, 8, 5, 6].

(ii) The system (1.12) is exactly controllable from the right (using $h_{2}$ or $h_{3}$ as control inputs with $h_{1} \equiv 0$ ), but only null controllable from the left (using $h_{1}$ as a control input with $h_{2}=h_{3} \equiv 0$ ). The system thus behaves like a parabolic system if control is acted only on the left end of the spatial domain and behaves like a hyperbolic system is control is allowed to act on the right end of the spatial domain [32, 13].

\section{$2 \quad$ Linear Systems}

Consideration is first given to the associate linear open loop control system

$$
\partial_{t} v+\partial_{x}^{3} v+\mu \partial_{x} v=G h, \quad v(x, 0)=v_{0}(x), \quad x \in \mathbb{T}, t \in \mathbb{R},
$$

where the operator $G$ is as defined in Section 1 and $h$ is the applied control function.

Let $A$ denote the operator

$$
A w=-w^{\prime \prime \prime}-\mu w^{\prime}
$$

with its domain $\mathcal{D}(A)=H^{3}(\mathbb{T})$. The operator $A$ generates a strongly continuous group $W(t)$ on the space $L^{2}(\mathbb{T})$; the eigenfunctions are simply the orthonormal Fourier basis functions in $L^{2}(\mathbb{T})$,

$$
\phi_{k}(x)=\frac{1}{\sqrt{2 \pi}} e^{i k x}, \quad k=0, \pm 1, \pm 2, \cdots
$$

The corresponding eigenvalue of $\phi_{k}$ is

$$
\lambda_{k}=i k^{3}-i \mu k, k=0, \pm 1, \pm 2, \cdots .
$$

For any $l \in \mathbb{Z}$, let

$$
m(l)=\#\left\{k \in \mathbb{Z} ; \lambda_{k}=\lambda_{l}\right\} .
$$

In addition, $A^{*}=-A, G^{*}=G$ and $W^{*}(-t)=W(t)$ for any $t \in \mathbb{R}$. Using the gap condition

$$
\lim _{|k| \rightarrow \infty}\left|\lambda_{k+1}-\lambda_{k}\right|=+\infty
$$

and the fact that $m(l) \leq 3$ for any $l$ and $m(l)=1$ for $|l|$ large enough, we may deduce from Ingham lemma that the system (2.1) is exactly controllable in $H_{0}^{s}(\mathbb{T})$ in small time for any $s \geq 0$. 
Theorem 2.1. [38, Theorem 2.1 and Corollary 2.1] Let $s \geq 0$ and $T>0$ be given. There exists a bounded linear operator

$$
\Phi: H_{0}^{s}(\mathbb{T}) \times H_{0}^{s}(\mathbb{T}) \mapsto L^{2}\left(0, T ; H_{0}^{s}(\mathbb{T})\right)
$$

such that for any $v_{0}, v_{1} \in H_{0}^{s}(\mathbb{T})$,

$$
W(T) v_{0}+\int_{0}^{T} W(T-t) G\left(\Phi\left(v_{0}, v_{1}\right)\right)(t) d t=v_{1}
$$

and

$$
\left\|\Phi\left(v_{0}, v_{1}\right)\right\|_{L^{2}\left(0, T ; H^{s}(\mathbb{T})\right)} \leq C\left(\left\|v_{0}\right\|_{s}+\left\|v_{1}\right\|_{s}\right)
$$

where $C>0$ depends only on $T$ and $\|g\|_{s}$.

The following estimate is a direct consequence of Theorem 2.1 ,

Corollary 2.2. Let $T>0$ be given. There exists $\delta>0$ such that

$$
\int_{0}^{T}\|G W(t) \phi\|_{0}^{2}(t) d t \geq \delta\|\phi\|_{0}^{2}
$$

for any $\phi \in L_{0}^{2}(\mathbb{T})$.

Note that the arguments presented in this paper give another proof of Corollary 2.2 .

In addition, if one chooses the following simple feedback law

$$
h(v)=-G^{*} v,
$$

the resulting closed-loop system

$$
\partial_{t} v+\partial_{x}^{3} v+\mu \partial_{x} v=-G G^{*} v, \quad v(x, 0)=v_{0}(x), \quad x \in \mathbb{T}
$$

is exponentially stable.

Proposition 2.3. Let $s \geq 0$ be given. There exists a number $\kappa>0$ independent of $s$ such that for any $v_{0} \in H_{0}^{s}(\mathbb{T})$, the corresponding solution $v$ of (2.2) satisfies

$$
\|v(., t)\|_{s} \leq C e^{-\kappa t}\left\|v_{0}\right\|_{s}
$$

for any $t \geq 0$ where $C>0$ is a constant depending only on $s$.

Proof. The case $s=0$ has been proved in [37, Theorem 2]. We only provide the proof for the case $s=3$. The case of $0<s<3$ follows by interpolation. The other cases of $s$ can be proved similarly.

Pick any $v_{0} \in H_{0}^{3}(\mathbb{T})$ and let $w=\partial_{t} v$. Then $w$ solves

$$
\partial_{t} w+\partial_{x}^{3} w+\mu \partial_{x} w=-G G^{*} w, \quad w(x, 0)=w_{0}(x), \quad x \in \mathbb{T}
$$

where $w_{0}(x)=-v_{0}^{\prime \prime \prime}(x)-\mu v_{0}^{\prime}-G G^{*} v_{0}(x)$ belongs to $L_{0}^{2}(\mathbb{T})$. Thus

$$
\|w(\cdot, t)\|_{0}\left\|\partial_{t} v(\cdot, t)\right\|_{0} \leq C_{0} e^{-\kappa t}\left\|w_{0}\right\|_{0}
$$

for any $t \geq 0$. From the equation

$$
\partial_{x}^{3} v+\mu \partial_{x} v=-w-G G^{*} v
$$

it follows that

$$
\|v(\cdot, t)\|_{3} \leq C_{3} e^{-\kappa t}\left\|v_{0}\right\|_{3}
$$

for any $t \geq 0$. The proof is complete. 
Next we show that it is possible to choose an appropriate linear feedback law such that the decay rate of the resulting closed-loop system is as large as one desires.

For given $\lambda>0$, define

$$
L_{\lambda} \phi=\int_{0}^{1} e^{-2 \lambda \tau} W(-\tau) G G^{*} W^{*}(-\tau) \phi d \tau
$$

for any $\phi \in H^{s}(\mathbb{T})$. Clearly, $L_{\lambda}$ is a bounded linear operator from $H^{s}(\mathbb{T})$ to $H^{s}(\mathbb{T})$. Moreover, $L_{\lambda}$ is a self-adjoint positive operator on $L_{0}^{2}(\mathbb{T})$, and so is its inverse $L_{\lambda}^{-1} . L_{\lambda}$ is therefore an isomorphism from $L_{0}^{2}(\mathbb{T})$ onto itself. The following result claims that the same is true on $H_{0}^{s}(\mathbb{T})$.

Lemma 2.4. $L_{\lambda}$ is an isomorphism from $H_{0}^{s}(\mathbb{T})$ onto $H_{0}^{s}(\mathbb{T})$ for all $s \geq 0$.

Proof. Since the result is known for $s=0$, and $L_{\lambda}$ maps $H_{0}^{s}(\mathbb{T})$ into itself, we only have to prove that for any $v \in L_{0}^{2}(\mathbb{T}), L_{\lambda} v \in H_{0}^{s}(\mathbb{T})$ implies $v \in H_{0}^{s}(\mathbb{T})$, i.e. $D^{s} v \in L^{2}(\mathbb{T})$. Using the continuity of $L_{\lambda}^{-1}$ on $L_{0}^{2}(\mathbb{T})$ and a commutator estimate similar to [20, Lemma A.1], we obtain

$$
\begin{aligned}
\left\|D^{s} v\right\|_{0} \leq & C\left\|L_{\lambda} D^{s} v\right\|_{0} \\
\leq & C\left\|\int_{0}^{1} e^{-2 \lambda \tau} W(-\tau) G G^{*} W^{*}(-\tau) D^{s} v d \tau\right\|_{0} \\
\leq & C\left\|D^{s} \int_{0}^{1} e^{-2 \lambda \tau} W(-\tau) G G^{*} W^{*}(-\tau) v d \tau\right\|_{0} \\
& \quad+C\left\|\int_{0}^{1} e^{-2 \lambda \tau} W(-\tau)\left[G G^{*}, D^{s}\right] W^{*}(-\tau) v d \tau\right\|_{0} \\
\leq & C\left\|L_{\lambda} v\right\|_{s}+C_{s}\|v\|_{s-1} .
\end{aligned}
$$

The result follows at once for $s \in[0,1]$. An induction yields the result for any $s \geq 0$.

Choose the feedback control

$$
h=-G^{*} L_{\lambda}^{-1} v .
$$

The resulting closed-loop system reads:

$$
\partial_{t} v+\partial_{x}^{3} v+\mu \partial_{x} v=-K_{\lambda} v, \quad v(x, 0)=v_{0}(x), x \in \mathbb{T},
$$

with

$$
K_{\lambda}:=G G^{*} L_{\lambda}^{-1}
$$

If $\lambda=0$, we define $K_{0}=G G^{*}$.

Proposition 2.5. Let $s \geq 0$ and $\lambda>0$ be given. For any $v_{0} \in H_{0}^{s}(\mathbb{T})$, the system (2.3) admits a unique solution $v \in C\left(\mathbb{R}^{+} ; H_{0}^{s}(\mathbb{T})\right)$. Moreover, there exists $M=M_{s}$ depending on s such that

$$
\|v(., t)\|_{s} \leq M_{s} e^{-\lambda t}\left\|v_{0}\right\|_{s}
$$

for any $t \geq 0$.

Proof. The case $s=0$ follows from [41, Theorem 2.1]. The other cases of $s$ are proved as for Proposition 2.3.

\section{Preliminaries}

In this section we present some results which are essential to establish the exact controllability and stabilizability of the nonlinear systems. 


\subsection{The Bourgain space and its properties.}

For given $b, s \in \mathbb{R}$, and a function $u: \mathbb{T} \times \mathbb{R} \rightarrow \mathbb{R}$, define the quantities

$$
\begin{aligned}
\|u\|_{X_{b, s}} & :=\left(\sum_{k=-\infty}^{\infty} \int_{\mathbb{R}}\langle k\rangle^{2 s}\left\langle\tau-k^{3}+\mu k\right\rangle^{2 b}|\widehat{\widehat{u}}(k, \tau)|^{2} d \tau\right)^{\frac{1}{2}}, \\
\|u\|_{Y_{b, s}} & :=\left(\sum_{k=-\infty}^{\infty}\left(\int_{\mathbb{R}}\langle k\rangle^{s}\left\langle\tau-k^{3}+\mu k\right\rangle^{b}|\widehat{\widehat{u}}(k, \tau)| d \tau\right)^{2}\right)^{\frac{1}{2}}
\end{aligned}
$$

where $\widehat{\widehat{u}}(k, \tau)$ denotes the Fourier transform of $u$ with respect to the space variable $x$ and the time variable $t$ (by contrast, $\widehat{u}(k, t)$ denotes the Fourier transform in space variable $x$ ) and $\langle\cdot\rangle=\sqrt{1+|\cdot|^{2}}$. Moreover, denote by $D^{r}$ the operator defined on $\mathcal{D}^{\prime}(\mathbb{T})$ by

$$
\begin{aligned}
\widehat{D^{r} u}(k) & =|k|^{r} \widehat{u}(k) & \text { if } \quad k \neq 0, \\
& =\widehat{u}(0) & \text { if } \quad k=0 .
\end{aligned}
$$

The Bourgain space $X_{b, s}$ (resp. $Y_{b, s}$ ) associated to the $\mathrm{KdV}$ equation on $\mathbb{T}$ is the completion of the space $\mathcal{S}(\mathbb{T} \times \mathbb{R})$ under the norm $\|u\|_{X_{b, s}}$ (resp. $\|u\|_{Y_{b, s}}$ ). Note that for any $u \in X_{b, s}$,

$$
\|u\|_{X_{b, s}}=\|W(-t) u\|_{H^{b}\left(\mathbb{R}, H^{s}(\mathbb{T})\right)} .
$$

For given $b, s \in \mathbb{R}$, let

$$
Z_{b, s}=X_{b, s} \cap Y_{b-\frac{1}{2}, s}
$$

be endowed with the norm

$$
\|u\|_{Z_{b, s}}\|u\|_{X_{b, s}}+\|u\|_{Y_{b-\frac{1}{2}, s}} .
$$

For a given interval $I$, let $X_{b, s}(I)$ (resp. $\left.Z_{b, s}(I)\right)$ be the restriction space of $X_{b, s}$ to the interval $I$ with the norm

$$
\begin{aligned}
\|u\|_{X_{b, s}(I)} & =\inf \left\{\|\widetilde{u}\|_{X_{b, s}} \mid \widetilde{u}=u \text { on } \mathbb{T} \times I\right\} \\
\text { (resp. }\|u\|_{Z_{b, s}(I)} & \left.=\inf \left\{\|\widetilde{u}\|_{Z_{b, s}} \mid \widetilde{u}=u \text { on } \mathbb{T} \times I\right\}\right) .
\end{aligned}
$$

For simplicity, we denote $X_{b, s}(I)$ (resp. $\left.Z_{b, s}(I)\right)$ by $X_{b, s}^{T}$ (resp. $Z_{b, s}^{T}$ ) if $I=(0, T)$. The following properties of the spaces $X_{b, s}^{T}$ are $Z_{b, s}^{T}$ are easily verified.

(i) $X_{b, s}(I)$ is a Hilbert space.

(ii) $D^{r} u \in X_{b, s-r}(I)$ for any $u \in X_{b, s}(I)$.

(iii) If $b_{1} \leq b_{2}$ and $s_{1} \leq s_{2}$, then $X_{b_{2}, s_{2}}$ is continuously imbedded in the space $X_{b_{1}, s_{1}}$.

(iv) For a given finite interval $I$, if $b_{1}<b_{2}$ and $s_{1}<s_{2}$, then the space $X_{b_{2}, s_{2}}(I)$ is compactly imbedded in the space $X_{b_{1}, s_{1}}(I)$.

(v) $Z_{\frac{1}{2}, s}(I) \subset C\left(\bar{I} ; H^{s}(\mathbb{T})\right)$ for any $s \in \mathbb{R}$.

Lemma 3.1. Let $b, s \in \mathbb{R}$ and $T>0$ be given. There exists a constant $C>0$ such that 
(i) for any $\phi \in H^{s}(\mathbb{T})$,

$$
\begin{aligned}
\|W(t) \phi\|_{X_{b, s}^{T}} & \leq C\|\phi\|_{s} \\
\|W(t) \phi\|_{Z_{b, s}^{T}} & \leq C\|\phi\|_{s}
\end{aligned}
$$

(ii) for any $f \in X_{b-1, s}^{T}$,

$$
\left\|\int_{0}^{t} W(t-\tau) f(\tau) d \tau\right\|_{X_{b, s}^{T}} \leq C\|f\|_{X_{b-1, s}^{T}}
$$

provided that $b>\frac{1}{2}$;

(iii) for any $f \in Z_{-\frac{1}{2}, s}^{T}$,

$$
\left\|\int_{0}^{t} W(t-\tau) f(\tau) d \tau\right\|_{Z_{\frac{1}{2}, s}^{T}} \leq C\|f\|_{Z_{-\frac{1}{2}, s}^{T}} .
$$

Proof. See e.g. [42] or [7].

Lemma 3.2. (Strichartz estimates) The following estimates hold:

$$
\begin{aligned}
\left\|\sum_{k, l \in \mathbb{Z}} c_{k, l} e^{i(k x+l t)}\right\|_{L^{4}\left(\mathbb{T}^{2}\right)} & \leq C\left(\sum_{k, l \in \mathbb{Z}}\left(1+\left|l-k^{3}+\mu k\right|\right)^{\frac{2}{3}}\left|c_{k, l}\right|^{2}\right)^{\frac{1}{2}}, \\
\|u\|_{L^{4}\left(\mathbb{T}^{2}\right)} & \leq C\|u\|_{X_{\frac{1}{3}, 0}}, \\
\|u\|_{L^{4}(\mathbb{T} \times(0, T))} & \leq C\|u\|_{X_{\frac{1}{3}, 0}^{T}} .
\end{aligned}
$$

Proof. (3.2) comes from [3, Proposition 7.15]. To prove (3.3), pick any $u \in X_{\frac{1}{3}, 0}$ decomposed as

$$
u(x, t)=\sum_{k \in \mathbb{Z}} \int_{\mathbb{R}} \widehat{\widehat{u}}(k, \tau) e^{i(k x+\tau t)} d \tau .
$$

Writing $\tau=l+\sigma$ with $l \in \mathbb{Z}, \sigma \in[0,1)$, we have that

$$
u(x, t)=\int_{0}^{1} e^{i \sigma t} \sum_{k, l \in \mathbb{Z}} \widehat{\widehat{u}}(k, l+\sigma) e^{i(k x+l t)} d \sigma .
$$

Using (3.2) and Cauchy-Schwarz, we obtain

$$
\begin{aligned}
\|u\|_{L^{4}\left(\mathbb{T}^{2}\right)} & \leq \int_{0}^{1}\left\|\sum_{k, l \in \mathbb{Z}} \widehat{\widehat{u}}(k, l+\sigma) e^{i(k x+l t)}\right\|_{L^{4}\left(\mathbb{T}^{2}\right)} d \sigma \\
& \leq C \int_{0}^{1}\left(\sum_{k, l \in \mathbb{Z}}\left(1+\left|l-k^{3}+\mu k\right|\right)^{\frac{2}{3}}|\widehat{\widehat{u}}(k, l+\sigma)|^{2}\right)^{\frac{1}{2}} d \sigma \\
& \leq C\left(\sum_{k \in \mathbb{Z}} \int_{0}^{1} \sum_{l \in \mathbb{Z}}\left(1+\left|l-k^{3}+\mu k\right|\right)^{\frac{2}{3}}|\widehat{\hat{u}}(k, l+\sigma)|^{2} d \sigma\right)^{\frac{1}{2}} \\
& \leq C\left(\left.\sum_{k \in \mathbb{Z}} \int_{\mathbb{R}}\left(1+\left|\tau-k^{3}+\mu k\right|\right)^{\frac{2}{3} \mid \widehat{u}}(k, \tau)\right|^{2} d \tau\right)^{\frac{1}{2}} .
\end{aligned}
$$


It remains to establish (3.4). Let $T>0$ and $u \in X_{\frac{1}{3}, 0}^{T}$. Pick $p \in \mathbb{N}^{*}$ with $T \leq 2 \pi p$, and an extension $\tilde{u} \in X_{\frac{1}{3}, 0}$ of $u$ with $\|\tilde{u}\|_{X_{\frac{1}{3}, 0}} \leq 2\|u\|_{X_{\frac{1}{3}, 0}^{T}}$. Then

$$
\|u\|_{L^{4}(\mathbb{T} \times(0, T))}^{4} \leq\|\tilde{u}\|_{L^{4}(\mathbb{T} \times(0,2 \pi p))}^{4} \leq p\left(C\|\tilde{u}\|_{X_{\frac{1}{3}, 0}}\right)^{4} \leq C^{\prime}\|u\|_{X_{\frac{1}{3}, 0}^{T}}^{4} .
$$

Note that $C^{\prime}$ depends only on $T$.

Lemma 3.3 (Bilinear estimates). Let $s \geq 0, T \in(0,1)$, and $u, v \in X_{\frac{1}{2}, s}^{T} \cap L^{2}\left(0, T ; L_{0}^{2}(\mathbb{T})\right)$. Then there exist some constants $\theta>0$ and $C>0$ independent of $T$ and $u, v$ such that

$$
\left\|(u v)_{x}\right\|_{Z_{-\frac{1}{2}, s}^{T}} \leq C T^{\theta}\|u\|_{X_{\frac{1}{2}, s}^{T}}\|v\|_{X_{\frac{1}{2}, s}^{T}}
$$

The proof of Lemma 3.3 can be found in [3] with $\theta=1 / 12$ (see also [7]).

To end this section, we prove a multiplication property of the Bourgain space $X_{b, s}^{T}$. If $\psi=\psi(t)$ is any $C^{\infty}$ function, then $\psi u \in X_{b, s}^{T}$ for any $u \in X_{b, s}^{T}$. However, if $\phi=\phi(x) \in C^{\infty}(\mathbb{T})$, then $\phi u$ may not belong to the space $X_{b, s}^{T}$ for $u \in X_{b, s}^{T}$. Some regularity in the index $b$ is lost due to the fact that the multiplication by a (smooth) function of $x$ does not keep the structure in time of the harmonics. This loss is, in fact, unavoidable. For instance, for $k \geq 1$, let $u_{k}=\psi(t) e^{i k x} e^{i\left(k^{3}-\mu k\right) t}$, where $\psi \in C_{0}^{\infty}(\mathbb{R})$ takes the value 1 on $[-1,1]$. The sequence $\left\{u_{k}\right\}$ is uniformly bounded in the space $X_{b, 0}$ for every $b \geq 0$. However, multiplying $u_{k}$ by $\phi(x)=e^{i x}$, we observe that $\left\|e^{i x} u_{k}\right\|_{X_{b, 0}} \approx k^{2 b}$.

The next lemma shows that this is the worst case.

Lemma 3.4. Let $-1 \leq b \leq 1, s \in \mathbb{R}$ and $\varphi \in C^{\infty}(\mathbb{T})$. Then, for any $u \in X_{b, s}, \varphi(x) u \in X_{b, s-2|b|}$. Similarly, the multiplication by $\varphi$ maps $X_{b, s}^{T}$ into $X_{b, s-2|b|}^{T}$.

Proof. We first consider the case of $b=0$ and $b=1$. The other cases of $b$ will be derived later by interpolation and duality.

For $b=0, X_{0, s}=L^{2}\left(\mathbb{R}, H^{s}(\mathbb{T})\right)$ and the result is obvious. For $b=1$, note that $u \in X_{1, s}$ if and only if

$$
u \in L^{2}\left(\mathbb{R}, H^{s}(\mathbb{T})\right) \text { and } \partial_{t} u+\partial_{x}^{3} u+\mu \partial_{x} u \in L^{2}\left(\mathbb{R}, H^{s}(\mathbb{T})\right)
$$

and that

$$
\|u\|_{X_{1, s}}^{2}=\|u\|_{L^{2}\left(\mathbb{R}, H^{s}(\mathbb{T})\right)}^{2}+\left\|\partial_{t} u+\partial_{x}^{3} u+\mu \partial_{x} u\right\|_{L^{2}\left(\mathbb{R}, H^{s}(\mathbb{T})\right)}^{2} .
$$

Thus,

$$
\begin{aligned}
\|\varphi(x) u\|_{X_{1, s-2}}^{2}= & \|\varphi u\|_{L^{2}\left(\mathbb{R}, H^{s-2}(\mathbb{T})\right)}^{2}+\left\|\partial_{t}(\varphi u)+\partial_{x}^{3}(\varphi u)+\mu \partial_{x}(\varphi u)\right\|_{L^{2}\left(\mathbb{R}, H^{s-2}(\mathbb{T})\right)}^{2} \\
\leq & C\left(\|u\|_{L^{2}\left(\mathbb{R}, H^{s-2}(\mathbb{T})\right)}^{2}+\left\|\varphi\left(\partial_{t} u+\partial_{x}^{3} u+\mu \partial_{x} u\right)\right\|_{L^{2}\left(\mathbb{R}, H^{s-2}(\mathbb{T})\right)}^{2}\right. \\
& \left.+\left\|\left[\varphi, \partial_{x}^{3}+\mu \partial_{x}\right] u\right\|_{L^{2}\left(\mathbb{R}, H^{s-2}(\mathbb{T})\right)}^{2}\right) \\
\leq & C\left(\|u\|_{L^{2}\left(\mathbb{R}, H^{s-2}(\mathbb{T})\right)}^{2}+\left\|\partial_{t} u+\partial_{x}^{3} u+\mu \partial_{x} u\right\|_{L^{2}\left(\mathbb{R}, H^{s-2}(\mathbb{T})\right)}^{2}+\|u\|_{L^{2}\left(\mathbb{R}, H^{s}(\mathbb{T})\right)}^{2}\right) \\
\leq & C\|u\|_{X_{1, s}}^{2} .
\end{aligned}
$$

Here, we have used the fact that

$$
\left[\varphi, \partial_{x}^{3}+\mu \partial_{x}\right]=-3\left(\partial_{x} \varphi\right) \partial_{x}^{2}-3\left(\partial_{x}^{2} \varphi\right) \partial_{x}-\partial_{x}^{3} \varphi-\mu \partial_{x} \varphi
$$


is a differential operator of order 2. To conclude, we prove that the $X_{b, s}$ spaces are in interpolation. First, using Fourier transform, $X_{b, s}$ may be viewed as the weighted $L^{2}$ space $L^{2}\left(\mathbb{R}_{\tau} \times \mathbb{Z}_{k},\langle k\rangle^{2 s}\langle\tau-\right.$ $\left.k^{3}+\mu k\right\rangle^{2 b} \lambda \otimes \delta$ ), where $\lambda$ is the Lebesgue measure on $\mathbb{R}$ and $\delta$ is the discrete measure on $\mathbb{Z}$. Then, we use the complex interpolation theorem of Stein-Weiss for weighted $L^{p}$ spaces (see [1, p. 114]): for $0<\theta<1$

$$
\left(X_{0, s}, X_{1, s^{\prime}}\right)_{[\theta]} \approx L^{2}\left(\mathbb{R} \times \mathbb{Z},\langle k\rangle^{2 s(1-\theta)+2 s^{\prime} \theta}\left\langle\tau-k^{3}+\mu k\right\rangle^{2 \theta} \mu \otimes \delta\right) \approx X_{\theta, s(1-\theta)+s^{\prime} \theta} .
$$

Since the multiplication by $\varphi$ maps $X_{0, s}$ into $X_{0, s}$ and $X_{1, s}$ into $X_{1, s-2}$, we conclude that for $0 \leq b \leq$ 1 , it maps $X_{b, s}=\left(X_{0, s}, X_{1, s}\right)_{[b]}$ into $\left(X_{0, s}, X_{1, s-2}\right)_{[b]}=X_{b, s-2 b}$, which yields the $2 b$ loss of regularity as announced.

Then, by duality, this also implies that for $0 \leq b \leq 1$, the multiplication by $\varphi(x)$ maps $X_{-b,-s+2 b}$ into $X_{-b,-s}$. As the number $s$ may take arbitrary values in $\mathbb{R}$, we also have the result for $-1 \leq b \leq 0$ with a loss of $-2 b=2|b|$.

To get the same result for the restriction spaces $X_{b, s}^{T}$, we write the estimate for an extension $\tilde{u}$ of $u$, which yields

$$
\|\varphi u\|_{X_{b, s-2|b|}^{T}} \leq\|\varphi \tilde{u}\|_{X_{b, s-2|b|}} \leq C\|\tilde{u}\|_{X_{b, s}} .
$$

Taking the infimum on all the $\tilde{u}$, we get the claimed result.

\subsection{Propagation of compactness and regularity}

In this subsection, we present some properties of propagation of compactness and regularity for the linear differential operator $L \partial_{t}+\partial_{x}^{3}+\mu \partial_{x}$ associated with the $\mathrm{KdV}$ equation. Those propagation properties will play a key role when studying the global stabilizability of the KdV equation.

Proposition 3.5. Let $T>0$ and $0 \leq b^{\prime} \leq b \leq 1$ be given (with $b>0$ ) and suppose that $u_{n} \in X_{b, 0}^{T}$ and $f_{n} \in X_{-b,-2+2 b}^{T}$ satisfy

$$
\partial_{t} u_{n}+\partial_{x}^{3} u_{n}+\mu \partial_{x} u_{n}=f_{n}
$$

for $n=1,2, \ldots$ Assume that there exists a constant $C>0$ such that

$$
\left\|u_{n}\right\|_{X_{b, 0}^{T}} \leq C \quad \text { for all } n \geq 1,
$$

and that

$$
\left\|u_{n}\right\|_{X_{-b,-2+2 b}^{T}}+\left\|f_{n}\right\|_{X_{-b,-2+2 b}^{T}}+\left\|u_{n}\right\|_{X_{-b^{\prime},-1+2 b^{\prime}}^{T}} \rightarrow 0 \text { as } n \rightarrow \infty .
$$

In addition, assume that for some nonempty open set $\omega \subset \mathbb{T}$ it holds

$$
u_{n} \rightarrow 0 \text { strongly in } L^{2}\left(0, T ; L^{2}(\omega)\right) .
$$

Then

$$
u_{n} \rightarrow 0 \text { strongly in } L_{l o c}^{2}\left((0, T) ; L^{2}(\mathbb{T})\right) .
$$

Proof. Pick $\varphi \in C^{\infty}(\mathbb{T})$ and $\psi \in C_{0}^{\infty}((0, T))$ real valued and set

$$
B=\varphi(x) D^{-2} \text { and } A=\psi(t) B .
$$

Then

$$
A^{*}=\psi(t) D^{-2} \varphi(x)
$$


For $\varepsilon>0$, let $A_{\varepsilon}=A e^{\varepsilon \partial_{x}^{2}}=\psi(t) B_{\varepsilon}$ be a regularization of $A$. Then

$$
\begin{aligned}
\alpha_{n, \varepsilon} & :=\left(\left[A_{\varepsilon}, L\right] u_{n}, u_{n}\right)_{L^{2}(\mathbb{T} \times(0, T))} \\
& =\left(\left[A_{\varepsilon}, \partial_{x}^{3}+\mu \partial_{x}\right] u_{n}, u_{n}\right)-\left(\psi^{\prime}(t) B_{\varepsilon} u_{n}, u_{n}\right) .
\end{aligned}
$$

On the other hand,

$$
\alpha_{n, \varepsilon}=\left(f_{n}, A_{\varepsilon}^{*} u_{n}\right)_{L^{2}(\mathbb{T} \times(0, T))}+\left(A_{\varepsilon} u_{n}, f_{n}\right)_{L^{2}(\mathbb{T} \times(0, T))}
$$

since $L u_{n}=f_{n}$ and $L^{*}=-L$. By Lemma 3.4,

$$
\begin{aligned}
\left|\left(f_{n}, A_{\varepsilon}^{*} u_{n}\right)_{L^{2}(\mathbb{T} \times(0, T))}\right| & \leq\left\|f_{n}\right\|_{X_{-b,-2+2 b}^{T}}\left\|A_{\varepsilon}^{*} u_{n}\right\|_{X_{b, 2-2 b}^{T}} \\
& \leq\left\|f_{n}\right\|_{X_{-b,-2+2 b}^{T}}\left\|u_{n}\right\|_{X_{b, 0}^{T}}
\end{aligned}
$$

Consequently,

$$
\lim _{n \rightarrow \infty} \sup _{0<\varepsilon \leq 1}\left|\left(f_{n}, A_{\varepsilon}^{*} u_{n}\right)_{L^{2}(\mathbb{T} \times(0, T))}\right|=0 .
$$

Similarly, we have

$$
\lim _{n \rightarrow \infty} \sup _{0<\varepsilon \leq 1}\left|\left(A_{\varepsilon} u_{n}, f_{n}\right)_{L^{2}(\mathbb{T} \times(0, T))}\right|=0
$$

and

$$
\lim _{n \rightarrow \infty} \sup _{0<\varepsilon \leq 1}\left|\left(\psi^{\prime}(t) B_{\varepsilon} u_{n}, u_{n}\right)\right|=0 .
$$

Thus

$$
\lim _{n \rightarrow \infty} \sup _{0<\varepsilon \leq 1}\left|\alpha_{n, \varepsilon}\right|=0
$$

and therefore

$$
\lim _{n \rightarrow \infty} \sup _{0<\varepsilon \leq 1}\left|\left(\left[A_{\varepsilon}, \partial_{x}^{3}+\mu \partial_{x}\right] u_{n}, u_{n}\right)\right|=0 .
$$

In particular,

$$
\lim _{n \rightarrow \infty}\left(\left[A, \partial_{x}^{3}+\mu \partial_{x}\right] u_{n}, u_{n}\right)_{L^{2}(\mathbb{T} \times(0, T))}=0 .
$$

As $D^{-2}$ commutes with $\partial_{x}$, we have

$$
\left[A, \partial_{x}^{3}+\mu \partial_{x}\right]=-3 \psi(t)\left(\partial_{x} \varphi\right) \partial_{x}^{2} D^{-2}-3 \psi(t)\left(\partial_{x}^{2} \varphi\right) \partial_{x} D^{-2}-\psi(t)\left(\partial_{x}^{3} \varphi+\mu \partial_{x} \varphi\right) D^{-2}
$$

Using the same argument as in (3.8), we get

$$
\left(\psi(t)\left(\partial_{x}^{3} \varphi+\mu \partial_{x} \varphi\right) D^{-2} u_{n}, u_{n}\right)_{L^{2}(\mathbb{T} \times(0, T))} \rightarrow 0 .
$$

However, for the second term, the loss of regularity is too large if we use the estimates with the same $b$. Using the index $b^{\prime}$ instead, we have

$$
\begin{aligned}
\left(\psi(t)\left(\partial_{x}^{2} \varphi\right) \partial_{x} D^{-2} u_{n}, u_{n}\right) & \leq\left\|\psi(t)\left(\partial_{x}^{2} \varphi\right) \partial_{x} D^{-2} u_{n}\right\|_{X_{b^{\prime}, 1-2 b^{\prime}}^{T}}\left\|u_{n}\right\|_{X_{-b^{\prime},-1+2 b^{\prime}}^{T}} \\
& \leq\left\|u_{n}\right\|_{X_{b^{\prime}, 0}^{T}}\left\|u_{n}\right\|_{X_{-b^{\prime},-1+2 b^{\prime}}^{T}}
\end{aligned}
$$


which tends to 0 as $n \rightarrow \infty$, by (3.6)-(3.7). Note that $-\partial_{x}^{2} D^{-2}$ is the orthogonal projection on the subspace of functions with $\widehat{u}(0)=0$. Using Rellich Theorem combined to the fact that $b>0$, we easily see that $\widehat{u_{n}}(0, t)$ tends to 0 in $L^{2}(0, T)$ (strongly), and hence

$$
\left(\psi(t)\left(\partial_{x} \varphi\right) \widehat{u_{n}}(0, t), u_{n}\right)_{L^{2}(\mathbb{T} \times(0, T))} \rightarrow 0 .
$$

We have thus proved that for any $\varphi \in C^{\infty}(\mathbb{T})$ and any $\psi \in C_{0}^{\infty}((0, T))$

$$
\left(\psi(t)\left(\partial_{x} \varphi\right) u_{n}, u_{n}\right)_{L^{2}(\mathbb{T} \times(0, T))} \rightarrow 0 .
$$

Note that a function $\phi \in C^{\infty}(\mathbb{T})$ can be written in the form $\partial_{x} \varphi$ for some function $\varphi \in C^{\infty}(\mathbb{T})$ if and only if $\int_{\mathbb{T}} \phi(x) d x=0$. Thus, for any $\chi \in C_{0}^{\infty}(\omega)$ and any $x_{0} \in \mathbb{T}, \phi(x) \chi(x)-\chi\left(x-x_{0}\right)$ can be written as $\phi=\partial_{x} \varphi$ for some $\varphi \in C^{\infty}(\mathbb{T})$.

Since $u_{n}$ is strongly convergent to 0 in $L^{2}\left(0, T ; L^{2}(\omega)\right)$,

$$
\lim _{n \rightarrow \infty}\left(\psi(t) \chi u_{n}, u_{n}\right)_{L^{2}(\mathbb{T} \times(0, T))}=0 .
$$

Therefore, for any $x_{0} \in \mathbb{T}$,

$$
\lim _{n \rightarrow \infty}\left(\psi(t) \chi\left(\cdot-x_{0}\right) u_{n}, u_{n}\right)_{L^{2}(\mathbb{T} \times(0, T))}=0 .
$$

The proof is then completed by constructing a partition of unity of $\mathbb{T}$ involving functions of the form $\chi_{i}\left(\cdot-x_{0}^{i}\right)$ with $\chi_{i} \in C_{0}^{\infty}(\omega)$ and $x_{0}^{i} \in \mathbb{T}$.

Next we investigate the propagation of regularity for the operator $L=\partial_{t}+\partial_{x}^{3}+\mu \partial_{x}$.

Proposition 3.6. Let $T>0,0 \leq b<1, r \in \mathbb{R}$ and $f \in X_{-b, r}^{T}$ be given. Let $u \in X_{b, r}^{T}$ be a solution of

$$
\partial_{t} u+\partial_{x}^{3} u+\mu \partial_{x} u=f .
$$

If there exists a nonempty open set $\omega$ of $\mathbb{T}$ such that $u \in L_{\text {loc }}^{2}\left((0, T), H^{r+\rho}(\omega)\right)$ for some $\rho$ with

$$
0<\rho \leq \min \left\{1-b, \frac{1}{2}\right\}
$$

then $u \in L_{\text {loc }}^{2}\left((0, T), H^{r+\rho}(\mathbb{T})\right)$.

Proof. Set $s=r+\rho$ and for $n=1,2, \ldots$

$$
u_{n}=e^{\frac{1}{n} \partial_{x}^{2}} u=: \Xi_{n} u, f_{n}=\Xi_{n} f=L u_{n} .
$$

There exists a constant $C>0$ such that

$$
\left\|u_{n}\right\|_{X_{b, r}^{T}} \leq C, \quad\left\|f_{n}\right\|_{X_{-b, r}^{T}} \leq C \quad \forall n \geq 1
$$

Pick $\varphi \in C^{\infty}(\mathbb{T})$ and $\psi \in C_{0}^{\infty}((0, T))$ as in the proof of Proposition 3.5, and set

$$
B=D^{2 s-2} \varphi(x) \text { and } A=\psi(t) B .
$$

We have

$$
\begin{aligned}
& \left(L u_{n}, A^{*} u_{n}\right)_{L^{2}(\mathbb{T} \times(0, T))}+\left(A u_{n}, L u_{n}\right)_{L^{2}(\mathbb{T} \times(0, T))} \\
= & \left(\left[A, \partial_{x}^{3}+\mu \partial_{x}\right] u_{n}, u_{n}\right)_{L^{2}(\mathbb{T} \times(0, T))}-\left(\psi^{\prime}(t) B u_{n}, u_{n}\right)
\end{aligned}
$$




$$
\begin{aligned}
\left|\left(A u_{n}, f_{n}\right)_{L^{2}(\mathbb{T} \times(0, T))}\right| & \leq\left\|A u_{n}\right\|_{X_{b,-r}^{T}}\left\|f_{n}\right\|_{X_{-b, r}^{T}} \\
& \leq\left\|u_{n}\right\|_{X_{b, r+2 \rho-2+2 b}^{T}}\left\|f_{n}\right\|_{X_{-b, r}^{T}} \\
& \leq C\left\|u_{n}\right\|_{X_{b, r}^{T}}\left\|f_{n}\right\|_{X_{-b, r}^{T}} \\
& \leq C
\end{aligned}
$$

since $r+2 \rho-2+2 b \leq r$. The same estimates for the other terms imply that

$$
\left|\left(\left[A, \partial_{x}^{3}+\mu \partial_{x}\right] u_{n}, u_{n}\right)_{L^{2}(\mathbb{T} \times(0, T))}\right| \leq C .
$$

Note that

$$
\left[A, \partial_{x}^{3}+\mu \partial_{x}\right]=-3 \psi(t) D^{2 s-2}\left(\partial_{x} \varphi\right) \partial_{x}^{2}-3 \psi(t) D^{2 s-2}\left(\partial_{x}^{2} \varphi\right) \partial_{x}-\psi(t) D^{2 s-2}\left(\partial_{x}^{3} \varphi+\mu \partial_{x} \varphi\right)
$$

and $2 s-2+1=2 r+2 \rho-1 \leq 2 r$. We have

$$
\begin{aligned}
& \left|\left(\psi(t) D^{2 s-2}\left(\partial_{x}^{2} \varphi\right) \partial_{x} u_{n}, u_{n}\right)_{L^{2}(\mathbb{T} \times(0, T))}\right| \\
& \quad \leq C\left\|\psi(t) D^{2 s-2}\left(\partial_{x}^{2} \varphi\right) \partial_{x} u_{n}\right\|_{L^{2}\left(0, T ; H^{-r}(\mathbb{T})\right)}\left\|u_{n}\right\|_{L^{2}\left(0, T ; H^{r}(\mathbb{T})\right)} \\
& \quad \leq C\left\|u_{n}\right\|_{L^{2}\left(0, T ; H^{r}(\mathbb{T})\right)}^{2} \\
& \quad \leq C
\end{aligned}
$$

and

$$
\begin{aligned}
& \left|\left(\psi(t) D^{2 s-2}\left(\partial_{x}^{3} \varphi+\mu \partial_{x} \varphi\right) u_{n}, u_{n}\right)_{L^{2}(\mathbb{T} \times(0, T))}\right| \\
& \quad \leq C\left\|\psi(t) D^{2 s-2}\left(\partial_{x}^{3} \varphi+\mu \partial_{x} \varphi\right) u_{n}\right\|_{L^{2}\left(0, T ; H^{-r}(\mathbb{T})\right)}\left\|u_{n}\right\|_{L^{2}\left(0, T ; H^{r}(\mathbb{T})\right)} \\
& \quad \leq C\left\|u_{n}\right\|_{L^{2}\left(0, T ; H^{r}(\mathbb{T})\right)}^{2} \\
& \quad \leq C
\end{aligned}
$$

for any $n \geq 1$. Thus

$$
\left|\left(\psi(t) D^{2 s-2}\left(\partial_{x} \varphi\right) \partial_{x}^{2} u_{n}, u_{n}\right)\right| \leq C
$$

For any $\chi \in C_{0}^{\infty}(\omega)$,

$$
\begin{aligned}
& \left(\psi(t) D^{2 s-2} \chi^{2} \partial_{x}^{2} u_{n}, u_{n}\right) \\
& =\left(\psi(t) D^{s-2} \chi \partial_{x}^{2} u_{n}, \chi D^{s} u_{n}\right)+\left(\psi(t)\left[D^{s-2}, \chi\right] \chi \partial_{x}^{2} u_{n}, D^{s} u_{n}\right) \\
& =\left(\psi(t) D^{s-2} \chi \partial_{x}^{2} u_{n}, D^{s} \chi u_{n}\right)+\left(\psi(t) D^{s-2} \chi \partial_{x}^{2} u_{n},\left[\chi, D^{s}\right] u_{n}\right) \\
& \quad+\left(\psi(t)\left[D^{s-2}, \chi\right] \chi \partial_{x}^{2} u_{n}, D^{s} u_{n}\right)=: I_{1}+I_{2}+I_{3} .
\end{aligned}
$$

We infer from the assumptions that $\chi u \in L_{\text {loc }}^{2}\left((0, T), H^{s}(\mathbb{T})\right)$ and that $\chi \partial_{x}^{2} u \in L_{\text {loc }}^{2}\left((0, T), H^{s-2}(\mathbb{T})\right)$. Thus

$$
\chi u_{n}=\Xi_{n} \chi u+\left[\chi, \Xi_{n}\right] u
$$

is uniformly bounded in $L_{l o c}^{2}\left((0, T), H^{s}(\mathbb{T})\right)$ by [20, Lemma A.3] and the fact that $s \leq r+1$. Applying the same argument to $\chi \partial_{x}^{2} u_{n}$, we obtain

$$
\left|I_{1}\right| \leq C
$$

It follows from [20, Lemma A.1] and the fact that $u \in L^{2}\left(0, T ; H^{r}(\mathbb{T})\right)$ that

$$
\begin{aligned}
\left|I_{2}\right| & \leq C\left\|D^{r-2} \chi \partial_{x}^{2} u_{n}\right\|_{L^{2}\left(0, T ; L^{2}(\mathbb{T})\right)}\left\|D^{\rho}\left[\chi, D^{s}\right] u_{n}\right\|_{L^{2}\left(0, T ; L^{2}(\mathbb{T})\right)} \\
& \leq C\left\|u_{n}\right\|_{L^{2}\left(0, T ; H^{r}(\mathbb{T})\right)}\left\|u_{n}\right\|_{L^{2}\left(0, T ; H^{s-1+\rho(T)}\right)} \leq C .
\end{aligned}
$$


A similar bound may be obtained for $\left|I_{3}\right|$. Consequently,

$$
\left|\left(\psi(t) D^{2 s-2} \chi^{2} \partial_{x}^{2} u_{n}, u_{n}\right)\right| \leq C
$$

for any $n \geq 1$. Then, using (3.9) with $\partial_{x} \varphi=\chi^{2}(x)-\chi^{2}\left(x-x_{0}\right)$ yields

$$
\left|\left(\psi(t) D^{2 s-2} \chi^{2}\left(\cdot-x_{0}\right) \partial_{x}^{2} u_{n}, u_{n}\right)\right| \leq C
$$

for any $n \geq 1$. Using a partition of unity as in the proof of Proposition 3.5, we obtain

$$
\left|\left(\psi(t) D^{2 s-2} \partial_{x}^{2} u, u\right)\right| \leq C,
$$

that is

$$
\int_{0}^{T} \psi(t)\left(\sum_{k \neq 0}|k|^{2 s}|\widehat{u}(k, t)|^{2}\right) d t \leq C
$$

The proof is thus complete.

Corollary 3.7. Let $u \in X_{\frac{1}{2}, 0}^{T}$ be a solution of

$$
\partial_{t} u+\partial_{x}^{3} u+\mu \partial_{x} u+u \partial_{x} u=0 \text { on } \mathbb{T} \times(0, T) .
$$

Assume that $u \in C^{\infty}(\omega \times(0, T))$, where $\omega$ is a nonempty open set in $\mathbb{T}$. Then $u \in C^{\infty}(\mathbb{T} \times(0, T))$.

Proof. Recall that the mean value $[u]$ is conserved. Changing $\mu$ into $\mu+[u]$ if needed, we may assume that $[u]=0$. We have $u \partial_{x} u \in X_{-\frac{1}{2}, 0}^{T}$ by Lemma 3.3. It follows from Proposition 3.6 that $u \in L_{\text {loc }}^{2}\left((0, T), H^{\frac{1}{2}}(\mathbb{T})\right)$. Choose $t_{0}$ such that $u\left(t_{0}\right) \in H^{\frac{1}{2}}(\mathbb{T})$. We can then solve (3.10) in $X_{\frac{1}{2}, \frac{1}{2}}^{T}$ with the initial data $u\left(t_{0}\right)$. By uniqueness of the solution in $X_{\frac{1}{2}, 0}^{T}$, we conclude that $u \in X_{\frac{1}{2}, \frac{1}{2}}^{T}$. An iterated application of Proposition 3.6 yields that $u \in L^{2}\left(0, T ; H^{r}(\mathbb{T})\right)$ for every $r \in \mathbb{R}$, and hence $u \in C^{\infty}(\mathbb{T} \times(0, T))$.

Corollary 3.8. Let $\omega$ be a nonempty open set in $\mathbb{T}$ and let $u \in X_{\frac{1}{2}, 0}^{T}$ be a solution of

$$
\left\{\begin{aligned}
\partial_{t} u+\partial_{x}^{3} u+\mu \partial_{x} u+u \partial_{x} u & =0 & & \text { on } \mathbb{T} \times(0, T) \\
u & =c & & \text { on } \omega \times(0, T)
\end{aligned}\right.
$$

where $c \in \mathbb{R}$ denotes some constant. Then $u(x, t)=c$ on $\mathbb{T} \times(0, T)$

Proof. Using Corollary 3.7, we infer that $u \in C^{\infty}(\mathbb{T} \times(0, T))$. It follows that $u \equiv c$ on $\mathbb{T} \times(0, T)$ by the unique continuation property for the $\mathrm{KdV}$ equation (see [40, 32]).

\section{Nonlinear systems}

In this section, we are concerned with the stability properties of the closed loop system

$$
\left\{\begin{array}{l}
\partial_{t} u+\partial_{x}^{3} u+\mu \partial_{x} u+u \partial_{x} u=-K_{\lambda} u, \quad x \in \mathbb{T}, 0<t<T, \\
u(x, 0)=u_{0}(x), \quad x \in \mathbb{T},
\end{array}\right.
$$

where $\lambda \geq 0$ is a given number and $u_{0} \in L_{0}^{2}(\mathbb{T})$.

We first check that the system is globally well-posed in the space $H_{0}^{s}(\mathbb{T})$ for any $s \geq 0$. 
Theorem 4.1. Let $\lambda \geq 0$ and $s \geq 0$ be given. Then for any $T>0$ and any $u_{0} \in H_{0}^{s}(\mathbb{T})$, there exists a unique solution $u \in Z_{\frac{1}{2}, s}^{T} \cap C\left([0, T] ; L_{0}^{2}(\mathbb{T})\right)$ of (4.1). Furthermore, the following estimate holds

$$
\|u\|_{Z_{\frac{1}{2}, s}^{T}} \leq \alpha_{T, s}\left(\left\|u_{0}\right\|_{0}\right)\left\|u_{0}\right\|_{s}
$$

where $\alpha_{T, s}: \mathbb{R}^{+} \rightarrow \mathbb{R}^{+}$is a nondecreasing continuous function depending only on $T$ and $s$.

Proof. We shall first establish the existence and uniqueness of a solution $u \in Z_{\frac{1}{2}, s}^{T} \cap L^{2}\left(0, T ; L_{0}^{2}(\mathbb{T})\right)$ of (4.1) for $T>0$ small enough. Then we shall show that $T$ can be taken as large as one wishes.

Let $u_{0} \in H_{0}^{s}(\mathbb{T})$. Rewrite system (4.1) in its integral form

$$
u(t)=W(t) u_{0}-\int_{0}^{t} W(t-\tau)\left(u \partial_{x} u\right)(\tau) d \tau-\int_{0}^{t} W(t-\tau)\left[K_{\lambda} u\right](\tau) d \tau
$$

where $W(t)=e^{-t\left(\partial_{x}^{3}+\mu \partial_{x}\right)}$. For given $u_{0}$, define the map

$$
\Gamma(v)=W(t) u_{0}-\int_{0}^{t} W(t-\tau)\left(v \partial_{x} v\right)(\tau) d \tau-\int_{0}^{t} W(t-\tau)\left[K_{\lambda} v\right](\tau) d \tau .
$$

The following estimate is needed.

Lemma 4.2. For any $\varepsilon>0$ there exists a positive constant $C(\varepsilon)$ such that

$$
\left\|\int_{0}^{t} W(t-\tau)\left[K_{\lambda} v\right](\tau) d \tau\right\|_{Z_{\frac{1}{2}, s}^{T}} \leq C(\varepsilon) T^{1-\varepsilon}\|v\|_{Z_{\frac{1}{2}, s}^{T}} .
$$

Proof of Lemma 4.2. Let $v \in Z_{\frac{1}{2}, s}^{T}$. Pick an extension of $v$ to $\mathbb{T} \times \mathbb{R}$, still denoted by $v$, and such that

$$
\|v\|_{Z_{\frac{1}{2}, s}} \leq 2\|v\|_{Z_{\frac{1}{2}, s}^{T}}
$$

Pick any $\eta \in C^{\infty}(\mathbb{R})$ with $\eta(t)=1$ for $|t| \leq 1$ and $\eta(t)=0$ for $|t| \geq 2$. By Lemma 3.1, it is clearly sufficient to prove that

$$
\left\|\eta^{2}(t / T) K_{\lambda} v\right\|_{Z_{-\frac{1}{2}, s}} \leq C T^{1-\varepsilon}\|v\|_{Z_{\frac{1}{2}, s}} .
$$

Let us first estimate $\left\|\eta^{2}(t / T) K_{\lambda} v\right\|_{X_{-\frac{1}{2}, s}}$. We have that

$$
\left\|\eta^{2}(t / T) K_{\lambda} v\right\|_{X_{-\frac{1}{2}, s}} \leq\left\|\eta^{2}(t / T) K_{\lambda} v\right\|_{X_{\frac{-1+\varepsilon}{2}, s}} \leq C T^{\frac{1-\varepsilon}{2}}\left\|\eta(t / T) K_{\lambda} v\right\|_{X_{0, s}} \leq C T^{1-\varepsilon}\|v\|_{X_{\frac{1}{2}, s}}
$$

where we used [42, Lemma 2.11] twice and Lemma 2.4. This yields also

$$
\left\|\eta^{2}(t / T) K_{\lambda} v\right\|_{Y_{-1, s}} \leq\left\|\eta^{2}(t / T) K_{\lambda} v\right\|_{X_{\frac{-1+\varepsilon}{2}, s}} \leq C T^{1-\varepsilon}\|v\|_{X_{\frac{1}{2}, s}}
$$

and (4.5) follows. The proof of Lemma 4.2 is complete.

It follows then from Lemmas 3.1, 3.3 and 4.2 that there exist some positive constants $\theta, C_{1}, C_{2}$ and $C_{3}$ such that

$$
\begin{aligned}
&\|\Gamma(v)\|_{Z_{\frac{1}{2}, s}^{T}} \leq C_{1}\left\|u_{0}\right\|_{s}+C_{2} T^{\theta}\|v\|_{Z_{\frac{1}{2}, s}^{T}}^{2}+C_{3} T^{1-\varepsilon}\|v\|_{Z_{\frac{1}{2}, s}^{T}} \\
&\left\|\Gamma\left(v_{1}\right)-\Gamma\left(v_{2}\right)\right\|_{Z_{\frac{1}{2}, s}^{T}} \leq C_{2} T^{\theta}\left\|v_{1}+v_{2}\right\|_{Z_{\frac{1}{2}, s}^{T}}\left\|v_{1}-v_{2}\right\|_{Z_{\frac{1}{2}, s}^{T}}+C_{3} T^{1-\varepsilon}\left\|v_{1}-v_{2}\right\|_{Z_{\frac{1}{2}, s}^{T}}
\end{aligned}
$$


for any $v, v_{1}, v_{2} \in Z_{\frac{1}{2}, s}^{T} \cap L^{2}\left(0, T ; L_{0}^{2}(\mathbb{T})\right)$. Pick $d=2 C_{1}\left\|u_{0}\right\|_{s}$ and $T>0$ such that

$$
2 C_{2} d T^{\theta}+C_{3} T^{1-\varepsilon} \leq \frac{1}{2}
$$

Then

$$
\|\Gamma(v)\|_{Z_{\frac{1}{2}, s}^{T}} \leq d
$$

and

$$
\left\|\Gamma\left(v_{1}\right)-\Gamma\left(v_{2}\right)\right\|_{Z_{\frac{1}{2}, s}^{T}} \leq \frac{1}{2}\left\|v_{1}-v_{2}\right\|_{Z_{\frac{1}{2}, s}^{T}}
$$

whenever $\|v\|_{Z_{\frac{1}{2}, s}^{T}} \leq d,\left\|v_{1}\right\|_{Z_{\frac{1}{2}, s}^{T}} \leq d$, and $\left\|v_{2}\right\|_{Z_{\frac{1}{2}, s}^{T}} \leq d$. Thus the map $\Gamma$ is a contraction in the closed ball $B_{d}(0)$ of $Z_{\frac{1}{2}, s}^{T} \cap L^{2}\left(0, T ; L_{0}^{2}(\mathbb{T})\right)$ for the $\|\cdot\|_{Z_{\frac{1}{2}, s}^{T}}$ norm. Its fixed point $u$ is the desired solution of (4.1) in the space $Z_{\frac{1}{2}, s}^{T} \cap L^{2}\left(0, T ; L_{0}^{2}(\mathbb{T})\right)$. It follows from the property (v) of the Bourgain space $Z_{b, s}^{T}$ recalled in the previous section that $u \in C\left([0, T] ; H_{0}^{s}(\mathbb{T})\right)$ with

$$
\|u\|_{L^{\infty}\left(0, T ; H^{s}(\mathbb{T})\right)} \leq C_{4}\|u\|_{Z_{\frac{1}{2}, s}^{T}} \leq 2 C_{1} C_{4}\left\|u_{0}\right\|_{s} .
$$

Let us now pass to the global existence of the solution. Assume first that $s=0$. The solution of (4.1) satisfies

$$
\|u(., t)\|_{0}^{2}=\|u\|_{0}^{2}-\int_{0}^{t}\left(G L_{\lambda}^{-1} u, G u\right)_{0}(\tau) d \tau \quad \forall t \geq 0
$$

which yields with Gronwall lemma

$$
\|u(., t)\|_{0}^{2} \leq\left\|u_{0}\right\|_{0}^{2} e^{C t}
$$

with $C=\|G\|^{2}\left\|L_{\lambda}^{-1}\right\|$. A standard continuation argument shows that (4.1) is globally well-posed in $L_{0}^{2}(\mathbb{T})$. (Note that $\|u(., t)\|_{0} \leq\left\|u_{0}\right\|_{0}$ when $\lambda=0$ and $t \geq 0$.) Next, we show that (4.1) is globally well-posed in the space $H_{0}^{3}(\mathbb{T})$. For a smooth solution $u$ of (4.1), let $v=u_{t}$. Then

$$
\left\{\begin{array}{l}
\partial_{t} v+\partial_{x}^{3} v+\mu \partial_{x} v+\partial_{x}(u v)=-K_{\lambda} v, \quad x \in \mathbb{T}, 0<t<T, \\
v(x, 0)=v_{0}(x), \quad x \in \mathbb{T},
\end{array}\right.
$$

where

$$
v_{0}=-K_{\lambda} u_{0}-u_{0}^{\prime \prime \prime}-\mu u_{0}^{\prime}-u_{0} u_{0}^{\prime} .
$$

For $T$ fulfilling (4.8), we have

$$
\|u\|_{Z_{\frac{1}{2}, 0}^{T}} \leq d=2 C_{1}\left\|u_{0}\right\|_{0}
$$

The same computations as those leading to (4.6) yield

$$
\|v\|_{Z_{\frac{1}{2}, 0}^{T}} \leq C_{1}\left\|v_{0}\right\|_{0}+\left(4 C_{1} C_{2} T^{\theta}\left\|u_{0}\right\|_{0}+C_{3} T^{1-\varepsilon}\right)\|v\|_{Z_{\frac{1}{2}, 0}^{T}}
$$

and hence

$$
\|v\|_{Z_{\frac{1}{2}, 0}^{T}} \leq 2 C_{1}\left\|v_{0}\right\|_{0}
$$

for $0<T<T_{1}\left(\left\|u_{0}\right\|_{0}\right)$, where $T_{1}(\cdot)$ is a continuous nonincreasing function. Therefore,

$$
\|v\|_{L^{\infty}\left(0, T ; L^{2}(\mathbb{T})\right)} \leq C_{4}\|v\|_{Z_{\frac{1}{2}, 0}^{T}} \leq C_{1}^{\prime}\left\|v_{0}\right\|_{0}
$$


for $0<T<T_{1}$ and $C_{1}^{\prime}=2 C_{1} C_{4}$. ¿From the equation

$$
\partial_{x}^{3} u=-K_{\lambda} u-v-\mu \partial_{x} u-u \partial_{x} u,
$$

we infer that for $0<t<T<T_{1}$

$$
\begin{aligned}
\left\|\partial_{x}^{3} u\right\|_{0} & \leq C_{7}\|u\|_{0}+\|v\|_{0}+\left(C_{8}+\|u\|_{0}\right)\left\|\partial_{x} u\right\|_{L_{x}^{\infty}} \\
& \leq C_{7}\|u\|_{0}+\|v\|_{0}+C_{9}\left(1+\|u\|_{0}\right)\|u\|_{0}^{\frac{1}{2}}\left\|\partial_{x}^{3} u\right\|_{0}^{\frac{1}{2}} \\
& \leq \frac{1}{2}\left\|\partial_{x}^{3} u\right\|_{0}+\|v\|_{0}+C_{10}\left(\|u\|_{0}+\|u\|_{0}^{3}\right) .
\end{aligned}
$$

Consequently,

$$
\|u\|_{L^{\infty}\left(0, T ; H^{3}(\mathbb{T})\right)} \leq \alpha_{T, 3}\left(\left\|u_{0}\right\|_{0}\right)\left\|u_{0}\right\|_{3}
$$

for $T<T_{1}\left(\left\|u_{0}\right\|_{0}\right)$. Combined to (4.9), this shows that $u \in C\left(\mathbb{R}^{+} ; H_{0}^{3}(\mathbb{T})\right)$ and that (4.2) holds true for $s=3$. A similar result can be obtained for any $s \in 3 \mathbb{N}^{*}$. For other values of $s$, the global well-posedness follows by nonlinear interpolation [43, 2]. The proof is complete.

Next we prove a local exponential stability result when applying the feedback law $h=-K_{\lambda} u$.

Theorem 4.3. Let $0<\lambda^{\prime}<\lambda$ and $s \geq 0$ be given. There exists $\delta>0$ such that for any $u_{0} \in H_{0}^{s}(\mathbb{T})$ with $\left\|u_{0}\right\|_{s} \leq \delta$, the corresponding solution $u$ of (4.1) satisfies

$$
\|u(., t)\|_{s} \leq C e^{-\lambda^{\prime} t}\left\|u_{0}\right\|_{s} \quad \text { for all } t \geq 0
$$

where $C>0$ is a constant independent of $u_{0}$.

Proof. We proceed as in [34, 36]. System (4.1) can be rewritten in an equivalent integral form

$$
u(t)=W_{\lambda}(t) u_{0}-\int_{0}^{t} W_{\lambda}(t-\tau)\left(u u_{x}\right)(\tau) d \tau
$$

where $W_{\lambda}(t)=e^{-t\left(\partial_{x}^{3}+\mu \partial_{x}+K_{\lambda}\right)}$. At this point we need to extend some estimates in Lemmas $3.1+3.3$ for the $C^{0}-$ group $W_{\lambda}(t)$.

Lemma 4.4. Let $s \geq 0, \lambda \geq 0$ and $T>0$ be given. Then there exists a constant $C>0$ such that (i) for any $\phi \in H_{0}^{s}(\mathbb{T})$

$$
\left\|W_{\lambda}(t) \phi\right\|_{Z_{\frac{1}{2}, s}^{T}} \leq C\|\phi\|_{s}
$$

(ii) For any $u, v \in Z_{\frac{1}{2}, s}^{T}$

$$
\left\|\int_{0}^{t} W_{\lambda}(t-\tau)(u v)_{x}(\tau) d \tau\right\|_{Z_{\frac{1}{2}, s}^{T}} \leq C\|u\|_{Z_{\frac{1}{2}, s}^{T}}\|v\|_{Z_{\frac{1}{2}, s}^{T}} .
$$

Proof of Lemma 4.4: An application of Duhamel formula gives

$$
W_{\lambda}(t) \phi=W(t) \phi-\int_{0}^{t} W(t-\tau)\left[K_{\lambda} W_{\lambda}(\tau) \phi\right] d \tau .
$$

Using Lemma 4.2, this yields

$$
\begin{aligned}
\left\|W_{\lambda}(t) \phi\right\|_{Z_{\frac{1}{2}, s}^{T}} & \leq\|W(t) \phi\|_{Z_{\frac{1}{2}, s}^{T}}+\left\|\int_{0}^{t} W(t-\tau)\left[K_{\lambda} W_{\lambda}(\tau) \phi\right] d \tau\right\|_{Z_{\frac{1}{2}, s}^{T}} \\
& \leq C\|\phi\|_{s}+C T^{1-\varepsilon}\left\|W_{\lambda}(t) \phi\right\|_{Z_{\frac{1}{2}, s}^{T}}
\end{aligned}
$$


(i) follows at once if $T$ is small enough, say $T<T_{0}$. For $T \geq T_{0}$, the result follows from an easy induction. To prove (ii), we use the identity

$$
\int_{0}^{t} W_{\lambda}(t-\tau) f(\tau) d \tau=\int_{0}^{t} W(t-\tau) f(\tau) d \tau-\int_{0}^{t} W(t-\tau) K_{\lambda}\left(\int_{0}^{\tau} W_{\lambda}(\tau-\sigma) f(\sigma) d \sigma\right) d \tau
$$

which gives with $f=(u v)_{x}$

$$
\begin{aligned}
& \left\|\int_{0}^{t} W_{\lambda}(t-\tau)(u v)_{x}(\tau) d \tau\right\|_{Z_{\frac{1}{2}, s}^{T}} \\
& \quad \leq\left\|\int_{0}^{t} W(t-\tau)(u v)_{x}(\tau) d \tau\right\|_{Z_{\frac{1}{2}, s}^{T}}+\left\|\int_{0}^{t} W(t-\tau) K_{\lambda}\left(\int_{0}^{\tau} W_{\lambda}(\tau-\sigma)(u v)_{x}(\sigma) d \sigma\right) d \tau\right\|_{Z_{\frac{1}{2}, s}^{T}} \\
& \quad \leq C\|u\|_{Z_{\frac{1}{2}, s}^{T}}\|v\|_{Z_{\frac{1}{2}, s}^{T}}+C T^{1-\varepsilon}\left\|\int_{0}^{t} W_{\lambda}(t-\tau)(u v)_{x}(\tau) d \tau\right\|_{Z_{\frac{1}{2}, s}^{T}}
\end{aligned}
$$

(ii) follows again if $T$ is small enough, say $T<T_{0}$. For $T \geq T_{0}$, the result follows from (i) and an easy induction.

For given $s \geq 0$, there exists by Proposition 2.5 some constant $C>0$ such that

$$
\left\|W_{\lambda}(t) u_{0}\right\|_{s} \leq C e^{-\lambda t}\left\|u_{0}\right\|_{s} \quad \forall t \geq 0
$$

Pick $T>0$ such that

$$
2 C e^{-\lambda T} \leq e^{-\lambda^{\prime} T}
$$

We seek a solution $u$ to the integral equation (4.11) as a fixed point of the map

$$
\Gamma(v)=W_{\lambda}(t) u_{0}-\int_{0}^{t} W_{\lambda}(t-\tau)\left(v v_{x}\right)(\tau) d \tau
$$

in some closed ball $B_{M}(0)$ in the space $Z_{\frac{1}{2}, s}^{T} \cap L^{2}\left(0, T ; L_{0}^{2}(\mathbb{T})\right)$ for the $\|v\|_{Z_{\frac{1}{2}, s}^{T}}$ norm. This will be done provided that $\left\|u_{0}\right\|_{s} \leq \delta$ where $\delta$ is a small number to be determined. Furthermore, to ensure the exponential stability with the claimed decay rate, the numbers $\delta$ and $M$ will be chosen in such a way that

$$
\|u(T)\|_{s} \leq e^{-\lambda^{\prime} T}\left\|u_{0}\right\|_{s} .
$$

By Lemma 4.4, there exist some positive constants $C_{1}, C_{2}$ (independent of $\delta$ and $M$ ) such that

$$
\|\Gamma(v)\|_{Z_{\frac{1}{2}, s}^{T}} \leq C_{1}\left\|u_{0}\right\|_{s}+C_{2}\|v\|_{Z_{\frac{1}{2}, s}^{T}}^{2}
$$

and

$$
\left\|\Gamma\left(v_{1}\right)-\Gamma\left(v_{2}\right)\right\|_{Z_{\frac{1}{2}, s}^{T}} \leq C_{2}\left\|v_{1}+v_{2}\right\|_{Z_{\frac{1}{2}, s}^{T}}\left\|v_{1}-v_{2}\right\|_{Z_{\frac{1}{2}, s}^{T}} .
$$

On the other hand, since $Z_{\frac{1}{2}, s}^{T} \subset C\left([0, T] ; H^{s}(\mathbb{T})\right)$, we have for some constant $C^{\prime}>0$ and all $v \in B_{M}(0)$

$$
\begin{aligned}
\|\Gamma(v)(T)\|_{s} & \leq\left\|W_{\lambda}(T) u_{0}\right\|_{s}+\left\|\int_{0}^{T} W_{\lambda}(T-t)\left(v v_{x}\right)(\tau) d \tau\right\|_{s} \\
& \leq C e^{-\lambda T} \delta+C^{\prime} M^{2} .
\end{aligned}
$$


Pick $\delta=C_{4} M^{2}$, where $C_{4}$ and $M$ are chosen so that

$$
\frac{C^{\prime}}{C_{4}} \leq C e^{-\lambda T}, \quad\left(C_{1} C_{4}+C_{2}\right) M^{2} \leq M, \quad \text { and } \quad 2 C_{2} M \leq \frac{1}{2}
$$

Then we have

$$
\begin{aligned}
\|\Gamma(v)\|_{Z_{\frac{1}{2}, s}^{T}} & \leq M \quad \forall v \in B_{M}(0) \\
\left\|\Gamma\left(v_{1}\right)-\Gamma\left(v_{2}\right)\right\|_{Z_{\frac{1}{2}, s}^{T}} & \leq \frac{1}{2}\left\|v_{1}-v_{2}\right\|_{Z_{\frac{1}{2}, s}^{T}} \quad \forall v_{1}, v_{2} \in B_{M}(0) .
\end{aligned}
$$

Therefore, $\Gamma$ is a contraction in $B_{M}(0)$. Furthermore, its unique fixed point $u \in B_{M}(0)$ fulfills

$$
\|u(T)\|_{s}=\|\Gamma(u)(T)\|_{s} \leq e^{-\lambda^{\prime} T} \delta
$$

Assume now that $0<\left\|u_{0}\right\|_{s}<\delta$. Changing $\delta$ into $\delta^{\prime}:=\left\|u_{0}\right\|_{s}$ and $M$ into $M^{\prime}=\left(\delta^{\prime} / \delta\right)^{\frac{1}{2}} M$, we infer that $\|u(T)\|_{s} \leq e^{-\lambda^{\prime} T}\left\|u_{0}\right\|_{s}$, and an obvious induction yields $\|u(n T)\|\left\|_{s} \leq e^{-\lambda^{\prime} n T}\right\| u_{0} \|_{s}$ for any $n \geq 0$. As $Z_{\frac{1}{2}, s}^{T} \cap L^{2}\left(0, T ; L_{0}^{2}(\mathbb{T})\right) \subset C\left([0, T] ; H_{0}^{s}(\mathbb{T})\right)$, we infer by the semigroup property that there exists some constant $C^{\prime}>0$ such that

$$
\|u(t)\|_{s} \leq C^{\prime} e^{-\lambda^{\prime} t}\left\|u_{0}\right\|_{s}
$$

provided that $\left\|u_{0}\right\|_{s} \leq \delta$. The proof is complete.

The stability result presented in Theorem 4.3 was local. We extend it to a global stability result in the following theorem.

Theorem 4.5. Assume $\lambda=0$ in 4.1. 3 There exists a $\kappa>0$ such that for any $R_{0}>0$, there exists a constant $C>0$ such that for any $u_{0} \in L_{0}^{2}(\mathbb{T})$ with

$$
\left\|u_{0}\right\|_{0} \leq R_{0}
$$

the corresponding solution $u$ of 4.1) (with $\lambda=0$ ) satisfies

$$
\|u(\cdot, t)\|_{0} \leq C e^{-\kappa t}\left\|u_{0}\right\|_{0} \quad \text { for all } t \geq 0 .
$$

Theorem 4.5 is a direct consequence of the following observability inequality.

Proposition 4.6. Let $T>0$ and $R_{0}>0$ be given. There exists a constant $\beta>1$ such that for any $u_{0} \in L_{0}^{2}(\mathbb{T})$ satisfying

$$
\left\|u_{0}\right\|_{0} \leq R_{0}
$$

the corresponding solution $u$ of (4.1) satisfies

$$
\left\|u_{0}\right\|_{0}^{2} \leq \beta \int_{0}^{T}\|G u\|_{0}^{2}(t) d t .
$$

Indeed, if (4.14) holds, then it follows from the energy estimate

$$
\|u(\cdot, t)\|_{0}^{2}=\left\|u_{0}\right\|_{0}^{2}-\int_{0}^{t}\|G u\|_{0}^{2}(\tau) d \tau \quad \forall t \geq 0
$$

\footnotetext{
${ }^{3}$ Recall that $K_{0}=G G^{*}$.
} 
that

$$
\|u(\cdot, T)\|_{0}^{2} \leq\left(1-\beta^{-1}\right)\left\|u_{0}\right\|_{0}^{2}
$$

Thus

$$
\|u(\cdot, m T)\|_{0}^{2} \leq\left(1-\beta^{-1}\right)^{m}\left\|u_{0}\right\|_{0}^{2}
$$

which gives (4.13) by the semigroup property. We obtain a constant $\kappa$ independent of $R_{0}$ by noticing that for $t>c\left(\left\|u_{0}\right\|_{0}\right)$, the $L^{2}$ norm of $u(., t)$ is smaller than 1 , so that we can take the $\kappa$ corresponding to $R_{0}=1$.

Now we present a proof of Proposition 4.6 .

Proof of Proposition 4.6: We prove the estimate (4.14) by contradiction. If (4.14) is not true, then for any $n \geq 1$, (4.1) admits a solution $u_{n} \in Z_{\frac{1}{2}, 0}^{T} \cap C\left([0, T] ; L_{0}^{2}(\mathbb{T})\right)$ satisfying

$$
\left\|u_{n}(0)\right\|_{0} \leq R_{0}
$$

and

$$
\int_{0}^{T}\left\|G u_{n}\right\|_{0}^{2} d t<\frac{1}{n}\left\|u_{0, n}\right\|_{0}^{2}
$$

where $u_{0, n}=u_{n}(0)$. Since $\alpha_{n}:=\left\|u_{0, n}\right\|_{0} \leq R_{0}$, one can choose a subsequence of $\left\{\alpha_{n}\right\}$, still denoted by $\left\{\alpha_{n}\right\}$, such that

$$
\lim _{n \rightarrow \infty} \alpha_{n}=\alpha .
$$

There are two possible cases: (i) $\alpha>0$ and (ii) $\alpha=0$.

(i) $\alpha>0$

Note that the sequence $\left\{u_{n}\right\}$ is bounded in both spaces $L^{\infty}\left(0, T ; L^{2}(\mathbb{T})\right)$ and $X_{\frac{1}{2}, 0}^{T}$. By Lemma 3.3. the sequence $\left\{\partial_{x}\left(u_{n}^{2}\right)\right\}$ is bounded in the space $X_{-\frac{1}{2}, 0}^{T}$. On the other hand, the space $X_{\frac{1}{2}, 0}^{T}$ is compactly imbedded in the space $X_{0,-1}^{T}$. Therefore, we can extract a subsequence of $\left\{u_{n}\right\}$, still denoted by $\left\{u_{n}\right\}$, such that

$$
\begin{aligned}
u_{n} & \rightarrow u \quad \text { weakly in } X_{\frac{1}{2}, 0}^{T}, \text { and strongly in } X_{0,-1}^{T}, \\
-\frac{1}{2} \partial_{x}\left(u_{n}^{2}\right) & \rightarrow f \quad \text { weakly in } X_{-\frac{1}{2}, 0}^{T},
\end{aligned}
$$

where $u \in X_{\frac{1}{2}, 0}^{T}$ and $f \in X_{-\frac{1}{2}, 0}^{T}$. Furthermore, since $X_{\frac{1}{2}, 0}^{T}$ is continuously imbedded in $L^{4}(\mathbb{T} \times(0, T))$ by (3.4), $u_{n}^{2}$ is bounded in $L^{2}(\mathbb{T} \times(0, T))$. It follows that $\partial_{x}\left(u_{n}^{2}\right)$ is bounded in

$$
L^{2}\left(0, T ; H^{-1}(\mathbb{T})\right)=X_{0,-1}^{T} .
$$

Conducting interpolation between $X_{-\frac{1}{2}, 0}^{T}$ and $X_{0,-1}^{T}$, we obtain that $\partial_{x}\left(u_{n}^{2}\right)$ is bounded in $X_{-\frac{1-\theta}{2},-\theta}^{T}=$ $X_{-\frac{1}{2}+\frac{\theta}{2},-\theta}^{T}$ for $\theta \in[0,1]$. As $X_{-\frac{1}{2}+\frac{\theta}{2},-\theta}^{T}$ is compactly imbedded in $X_{-\frac{1}{2},-1}^{T}$ for $0<\theta<1$, we can extract a subsequence of $\left\{u_{n}\right\}$, still denoted by $\left\{u_{n}\right\}$, such that $-\frac{1}{2} \partial_{x}\left(u_{n}^{2}\right)$ converges to $f$ strongly in $X_{-\frac{1}{2},-1}^{T}$. It follows from (4.16) that

$$
\int_{0}^{T}\left\|G u_{n}\right\|_{0}^{2} d t \longrightarrow \int_{0}^{T}\|G u\|_{0}^{2} d t=0
$$


which implies that $u(x, t)=c(t)$ on $\omega \times(0, T)$ for some function $c(t)$. Thus, passing to the limit in (4.1), we obtain

$$
\left\{\begin{aligned}
\partial_{t} u+\partial_{x}^{3} u+\mu \partial_{x} u & =f & \text { on } \mathbb{T} \times(0, T) \\
u & =c(t) & \text { on } \omega \times(0, T) .
\end{aligned}\right.
$$

Let $w_{n}=u_{n}-u$ and $f_{n}=-\frac{1}{2} \partial_{x}\left(u_{n}^{2}\right)-f-K_{0} u_{n}$. Note first that

$$
\int_{0}^{T}\left\|G w_{n}\right\|_{0}^{2} d t \int_{0}^{T}\left\|G u_{n}\right\|_{0}^{2} d t+\int_{0}^{T}\|G u\|_{0}^{2} d t-2 \int_{0}^{T}\left(G u_{n}, G u\right)_{0} d t \rightarrow 0
$$

Since $w_{n} \rightarrow 0$ weakly in $X_{\frac{1}{2}, 0}^{T}$, we infer from Rellich theorem that $\int_{\mathbb{T}} g(y) w_{n}(y, t) d y \rightarrow 0$ strongly in $L^{2}(0, T)$. Combined to (4.18), this yields

$$
\int_{0}^{T} \int_{\mathbb{T}} g(x)^{2} w_{n}(x, t)^{2} d x d t \rightarrow 0
$$

Thus

$$
\partial_{t} w_{n}+\partial_{x}^{3} w_{n}+\mu \partial_{x} w_{n}=f_{n}
$$

and

$$
f_{n} \underset{X_{-\frac{1}{2},-1}^{T}}{\longrightarrow} 0, \quad w_{n} \underset{L^{2}\left(0, T ; L^{2}(\tilde{\omega})\right)}{\longrightarrow} 0
$$

where $\tilde{\omega}:=\left\{g>\|g\|_{L^{\infty}(\mathbb{T})} / 2\right\}$.

Applying Proposition 3.5 with $b=\frac{1}{2}$ and $b^{\prime}=0$ yields that

$$
w_{n} \underset{L_{l o c}^{2}\left((0, T) ; L^{2}(\mathbb{T})\right)}{\longrightarrow} 0 .
$$

Consequently, $u_{n}^{2}$ tends to $u^{2}$ in $L_{l o c}^{1}\left((0, T) ; L^{1}(\mathbb{T})\right)$ and $\partial_{x}\left(u_{n}^{2}\right)$ tends to $\partial_{x}\left(u^{2}\right)$ in the distributional sense. Therefore $f=-\frac{1}{2} \partial_{x}\left(u^{2}\right)$ and $u \in X_{\frac{1}{2}, 0}^{T}$ satisfies

$$
\left\{\begin{array}{rlr}
\partial_{t} u+\partial_{x}^{3} u+\mu \partial_{x} u+\frac{1}{2} \partial_{x}\left(u^{2}\right) & =0 \quad \text { on } \mathbb{T} \times(0, T) \\
u & =c(t) \quad \text { on } \omega \times(0, T) .
\end{array}\right.
$$

The first equation gives $c^{\prime}(t)=0$ which, combined to Corollary 3.8, yields that $u(x, t) \equiv c$ for some constant $c \in \mathbb{R}$. Since $[u]=0, c=0$, and $u_{n}$ converges strongly to 0 in $L_{l o c}^{2}\left((0, T), L^{2}(\mathbb{T})\right)$. We can pick some time $t_{0} \in[0, T]$ such that $u_{n}\left(t_{0}\right)$ tends to 0 strongly in $L^{2}(\mathbb{T})$. Since

$$
\left\|u_{n}(0)\right\|_{0}^{2}=\left\|u_{n}\left(t_{0}\right)\right\|_{0}^{2}+\int_{0}^{t_{0}}\left\|G u_{n}\right\|_{0}^{2} d t
$$

it is inferred that $\alpha_{n}=\left\|u_{n}(0)\right\|_{0} \rightarrow 0$ which is a contradiction to the assumption $\alpha>0$.

(ii) $\alpha=0$.

Note first that $\alpha_{n}>0$ for all $n$. Set $v_{n}=u_{n} / \alpha_{n}$ for all $n \geq 1$. Then

$$
\partial_{t} v_{n}+\partial_{x}^{3} v_{n}+\mu \partial_{x} v_{n}+K_{0} v_{n}+\frac{\alpha_{n}}{2} \partial_{x}\left(v_{n}^{2}\right)=0
$$

and

$$
\int_{0}^{T}\left\|G v_{n}\right\|_{0}^{2} d t<\frac{1}{n}
$$


Because of

$$
\left\|v_{n}(0)\right\|_{0}=1
$$

the sequence $\left\{v_{n}\right\}$ is bounded in both spaces $L^{\infty}\left(0, T ; L^{2}(\mathbb{T})\right)$ and $X_{\frac{1}{2}, 0}^{T}$. Indeed, $\left\|v_{n}(t)\right\|_{0}$ is a nonincreasing function of $t$, and the boundedness of $\left\|v_{n}\right\|_{X_{\frac{1}{2}, 0}^{T}}$ for small values of $T$ follows from an estimate similar to (4.6) (since $\alpha_{n}$ is bounded). We can extract a subsequence of $\left\{v_{n}\right\}$, still denoted by $\left\{v_{n}\right\}$, such that $v_{n} \rightarrow v$ weakly in the space $X_{\frac{1}{2}, 0}^{T}$ and strongly in the spaces $X_{-\frac{1}{2},-1}^{T}$ and $X_{0,-1}^{T}$. Moreover, the sequence $\left\{\partial_{x}\left(v_{n}^{2}\right)\right\}$ is bounded in the space $X_{-\frac{1}{2}, 0}^{T}$, and therefore $\alpha_{n} \partial_{x}\left(v_{n}^{2}\right)$ tends to 0 in the space $X_{-\frac{1}{2}, 0}^{T}$. Finally, $\int_{0}^{T}\|G v\|_{0}^{2} d t=0$. Thus, $v$ solves

$$
\left\{\begin{aligned}
\partial_{t} v+\partial_{x}^{3} v+\mu \partial_{x} v & =0 & & \text { on } \mathbb{T} \times(0, T) \\
v & =c(t) & & \text { on } \omega \times(0, T) .
\end{aligned}\right.
$$

We infer that $v(x, t)=c(t)=c$ thanks to Holmgren Theorem, and that $c=0$ because of $[v]=0$.

According to (4.19)

$$
\int_{0}^{T}\left\|G v_{n}\right\|_{0}^{2} d t \longrightarrow 0
$$

and so $K_{0} v_{n}$ converges strongly to 0 in $X_{-\frac{1}{2},-1}^{T}$. Then, an application of Proposition 3.5 as in (i) shows that $v_{n}$ converges to 0 in $L_{l o c}^{2}\left((0, T), L^{2}(\mathbb{T})\right)$. Thus we can pick a time $t_{0} \in(0, T)$ such that $v_{n}\left(t_{0}\right)$ converges to 0 strongly in $L^{2}(\mathbb{T})$. Since

$$
\left\|v_{n}(0)\right\|_{0}^{2}=\left\|v_{n}\left(t_{0}\right)\right\|_{0}^{2}+\int_{0}^{t_{0}}\left\|G v_{n}\right\|_{0}^{2} d t
$$

we infer from (4.19) that $\left\|v_{n}(0)\right\|_{0} \rightarrow 0$ which is a contradiction to (4.20). The proof is complete.

Next we show that the solution $u$ of (4.1) (with $\lambda=0$ ) decays exponentially in any space $H^{s}(\mathbb{T})$.

Theorem 4.7. Assume that $\lambda=0$ in (4.1), and let $\kappa>0$ be the infimum of the numbers $\kappa$ given respectively in Proposition 2.3 and in Theorem 4.5. Let $s \geq 0$ and let $\kappa^{\prime} \in(0, \kappa)$ be given. Then there exists a nondecreasing continuous function $\alpha_{s, \kappa^{\prime}}: \mathbb{R}^{+} \rightarrow \mathbb{R}^{+}$such that for any $u_{0} \in H_{0}^{s}(\mathbb{T})$, the corresponding solution $u$ of 4.1) satisfies

$$
\|u(\cdot, t)\|_{s} \leq \alpha_{s, \kappa^{\prime}}\left(\left\|u_{0}\right\|_{0}\right) e^{-\kappa^{\prime} t}\left\|u_{0}\right\|_{s}
$$

for all $t \geq 0$.

Proof. The result for $s=0$ has already been established in Theorem 4.5 with $\kappa^{\prime}=\kappa$. Let us consider now the case $s=3$. Pick any number $R_{0}>0$ and any $u_{0} \in H_{0}^{3}(\mathbb{T})$ with $\left\|u_{0}\right\|_{0} \leq R_{0}$. Let $u$ denote the solution of (4.1) emanating from $u_{0}$ at $t=0$, and let $v=u_{t}$. Then $v$ solves

$$
\partial_{t} v+\partial_{x}^{3} v+\mu \partial_{x} v+\partial_{x}(u v)=-K_{0} v, v(x, 0)=v_{0}(x), x \in \mathbb{T}, t>0,
$$

where $v_{0}=-K_{0} u_{0}-\mu u_{0}^{\prime}-u_{0} u_{0}^{\prime}-u_{0}^{\prime \prime \prime}$. According to (4.2) and (4.13), for any $T>0$ there exists a number $C>0$ depending only on $R_{0}$ and $T$ such that

$$
\|u(\cdot, t)\|_{Z_{\frac{1}{2}, 0}^{[t, t+T]}} \leq C e^{-\kappa t}\left\|u_{0}\right\|_{0} \quad \text { for all } t \geq 0 .
$$


Thus, for any $\epsilon>0$, there exists a $t^{*}>0$ such that if $t \geq t^{*}$, one has

$$
\|u(\cdot, t)\|_{Z_{\frac{1}{2}, 0}^{[t, t+T]}} \leq \epsilon .
$$

At this point we need an exponential stability result for the linearized system

$$
\partial_{t} w+\partial_{x}^{3} w+\mu \partial_{x} w+\partial_{x}(a w)=-K_{0} w, w(x, 0)=w_{0}(x), x \in \mathbb{T}, t>0
$$

where $a \in Z_{\frac{1}{2}, s}^{T} \cap L^{2}\left(0, T ; L_{0}^{2}(\mathbb{T})\right)$ is a given function.

Lemma 4.8. Let $s \geq 0$ and $a \in Z_{\frac{1}{2}, s}^{T} \cap L^{2}\left(0, T ; L_{0}^{2}(\mathbb{T})\right)$ for all $T>0$. Then for any $\kappa^{\prime} \in(0, \kappa)$ there exist $T>0, \beta>0$ such that if

$$
\sup _{n \geq 1}\|a\|_{Z_{\frac{1}{2}, s}^{[n T,(n+1) T]}} \leq \beta
$$

then

$$
\|w(\cdot, t)\|_{s} \leq C e^{-\kappa^{\prime} t}\left\|w_{0}\right\|_{s} \quad \text { for all } t \geq 0,
$$

where $C>0$ is a constant independent of $w_{0}$.

Proof of Lemma 4.8: First, a proof similar to those of Theorem 4.1 shows that for any $T>0$ and any $s \geq 0$, if $a \in Z_{\frac{1}{2}, s}^{T} \cap L^{2}\left(0, T ; L_{0}^{2}(\mathbb{T})\right)$, then (4.23) admits a unique solution $w \in Z_{\frac{1}{2}, s}^{T} \cap L^{2}\left(0, T ; L_{0}^{2}(\mathbb{T})\right)$ and

$$
\|w\|_{Z_{\frac{1}{2}, s}^{T}} \leq \mu\left(\|a\|_{Z_{\frac{1}{2}, s}^{T}}\right)\left\|w_{0}\right\|_{s}
$$

where $\mu: \mathbb{R}^{+} \rightarrow \mathbb{R}^{+}$is a nondecreasing continuous function. Rewrite (4.23) in its integral form

$$
w(t)=W_{0}(t) w_{0}-\int_{0}^{t} W_{0}(t-\tau) \partial_{x}(a w)(\tau) d \tau
$$

where $W_{0}(t)=e^{-t\left(\partial_{x}^{3}+\mu \partial_{x}+K_{0}\right)}$. Thus, for any $T>0$, by Proposition 2.3, Lemma 4.4 and (4.24),

$$
\begin{aligned}
\|w(\cdot, T)\|_{s} & \leq C_{1} e^{-\kappa T}\left\|w_{0}\right\|_{s}+C_{2}\|a\|_{Z_{\frac{1}{2}, s}^{T}}\|w\|_{Z_{\frac{1}{2}, s}^{T}} \\
& \leq C_{1} e^{-\kappa T}\left\|w_{0}\right\|_{s}+C_{2}\|a\|_{Z_{\frac{1}{2}, s}^{T}} \mu\left(\|a\|_{Z_{\frac{1}{2}, s}^{T}}\right)\left\|w_{0}\right\|_{s}
\end{aligned}
$$

where $C_{1}>0$ is independent of $T$ while $C_{2}$ may depend on $T$. Let

$$
y_{n}=w(\cdot, n T) \quad \text { for } \quad n=1,2, \ldots
$$

Then, using the semigroup property of the system (4.23),

$$
\left\|y_{n+1}\right\|_{s} \leq C_{1} e^{-\kappa T}\left\|y_{n}\right\|_{s}+C_{2}\|a\|_{Z_{\frac{1}{2}, s}^{[n T,(n+1) T]}} \mu\left(\|a\|_{Z_{\frac{1}{2}, s}^{[n T,(n+1) T]}}\right)\left\|y_{n}\right\|_{s}
$$

for $n \geq 1$. Choose $T>0$ large enough and $\beta>0$ small enough so that

$$
C_{1} e^{-\kappa T}+C_{2} \beta \mu(\beta)=e^{-\kappa^{\prime} T}
$$

Then

$$
\left\|y_{n+1}\right\|_{s} \leq e^{-\kappa^{\prime} T}\left\|y_{n}\right\|_{s}
$$


for any $n \geq 1$ as long as

$$
\sup _{n \geq 1}\|a\|_{Z_{\frac{1}{2}, s}^{[n T,(n+1) T]}} \leq \beta .
$$

Thus

$$
\left\|y_{n}\right\|_{s} \leq e^{-n \kappa^{\prime} T}\left\|y_{0}\right\|_{s}
$$

for any $n \geq 1$, which implies that

$$
\|w(\cdot, t)\|_{s} \leq C e^{-\kappa^{\prime} t}\left\|w_{0}\right\|_{s}
$$

for all $t \geq 0$. The proof is complete.

Choose $\epsilon<\beta$, and then apply Lemma 4.8 to (4.22) to obtain

$$
\|v(\cdot, t)\|_{0} \leq C e^{-\kappa^{\prime}\left(t-t^{*}\right)}\left\|v\left(\cdot, t^{*}\right)\right\|_{0}
$$

for any $t \geq t^{*}$, or

$$
\|v(\cdot, t)\|_{0} \leq C_{1} e^{-\kappa^{\prime} t}\left\|v_{0}\right\|_{0}
$$

for any $t \geq 0$, where $C_{1}>0$ depends only on $R_{0}$. It then follows from the equation

$$
\partial_{x}^{3} u=-K_{0} u-u \partial_{x} u-\mu \partial_{x} u-v
$$

and Theorem 4.5 that

$$
\|u(\cdot, t)\|_{3} \leq C e^{-\kappa^{\prime} t}\left\|u_{0}\right\|_{3}
$$

for any $t \geq 0$, where $C>0$ depends only on $R_{0}$.

Thus the theorem has been proved for $s=0$ and $s=3$. Using the same argument for $u_{1}-u_{2}$ and $a=u_{1}+u_{2}$ for two different solutions $u_{1}$ and $u_{2}$, we obtain the Lipchitz stability estimate needed for interpolation:

$$
\left\|\left(u_{1}-u_{2}\right)(\cdot, t)\right\|_{0} \leq C e^{-\kappa^{\prime} t}\left\|\left(u_{1}-u_{2}\right)(\cdot, 0)\right\|_{0} .
$$

The case of $0<s<3$ follows by interpolation. The other cases can be proved similarly.

\section{Time-varying feedback law}

In this section we prove that it is possible to design a smooth time-varying feedback law ensuring a semiglobal stabilization with an arbitrary large decay rate.

Let $\lambda>0$ and $s \geq 0$ be given. According to Theorem 4.7, there exists a number $\kappa>0$ and a nondecreasing function $\alpha_{s}$ such that any solution $u$ of

$$
\partial_{t} u+\partial_{x}^{3} u+\mu \partial_{x} u+u \partial_{x} u=-G G^{*} u
$$

emanating from $u_{0} \in H_{0}^{s}(\mathbb{T})$ at $t=0$ fulfills

$$
\|u(t)\|_{s} \leq \alpha_{s}\left(\left\|u_{0}\right\|_{0}\right) e^{-\kappa t}\left\|u_{0}\right\|_{s} .
$$

On the other hand, it follows from Theorem 4.3 that for any fixed $\lambda^{\prime} \in(0, \lambda)$, any solution $u$ of

$$
\partial_{t} u+\partial_{x}^{3} u+\mu \partial_{x} u+u \partial_{x} u=-K_{\lambda} u
$$

emanating from $u_{0} \in H_{0}^{s}(\mathbb{T})$ at $t=0$ fulfills

$$
\|u(t)\|_{s} \leq C_{s} e^{-\lambda^{\prime} t}\left\|u_{0}\right\|_{s}
$$


provided that $\left\|u_{0}\right\|_{s} \leq r_{0}$, for some constant $C_{s}$ and some number $r_{0} \in(0,1)$. Pick any function $\theta \in C^{\infty}(\mathbb{R} ;[0,1])$ fulfilling the following properties:

$$
\begin{aligned}
& \theta(t)=1 \quad \text { for } \delta \leq t \leq 1-\delta \\
& \theta(t)=0 \quad \text { for } 1 \leq t \leq 2 \\
& \theta(t+2)=\theta(t) \quad \text { for all } t \in \mathbb{R}
\end{aligned}
$$

where $\delta \in(0,1 / 10)$ is a number whose value will be specified later. Pick a function $\rho \in C^{\infty}\left(\mathbb{R}^{+} ;[0,1]\right)$ such that

$$
\rho(r)=1 \quad \text { for } r \leq r_{0}, \quad \rho(r)=0 \quad \text { for } r \geq 1 .
$$

Let $T>0$ be given. We consider the following time-varying feedback law

$$
\begin{aligned}
K(u, t) & =\rho\left(\|u\|_{s}^{2}\right)\left[\theta\left(\frac{t}{T}\right) K_{\lambda} u+\theta\left(\frac{t-T}{T}\right) G G^{*} u\right]+\left(1-\rho\left(\|u\|_{s}^{2}\right)\right) G G^{*} u \\
& =G G^{*}\left\{\rho\left(\|u\|_{s}^{2}\right)\left[\theta\left(\frac{t}{T}\right) L_{\lambda}^{-1} u+\theta\left(\frac{t-T}{T}\right) u\right]+\left(1-\rho\left(\|u\|_{s}^{2}\right)\right) u\right\}
\end{aligned}
$$

The following result indicates that a semiglobal stabilization with an arbitrary decay rate can be obtained.

Theorem 5.1. Let $\lambda>0$ and let $K=K(u, t)$ be as given in (5.9). Pick any $\lambda^{\prime} \in(0, \lambda)$ and any $\lambda^{\prime \prime} \in\left(\lambda^{\prime} / 2,\left(\lambda^{\prime}+\kappa\right) / 2\right)$. Then there exists a time $T_{0}>0$ such that for $T>T_{0}, t_{0} \in \mathbb{R}$ and $u_{0} \in H_{0}^{s}(\mathbb{T})$, the unique solution of the closed-loop system

$$
\partial_{t} u+\partial_{x}^{3} u+\mu \partial_{x} u+u \partial_{x} u=-K(u, t), \quad u\left(t_{0}\right)=u_{0}
$$

satisfies

$$
\|u(., t)\|_{s} \leq \gamma_{s}\left(\left\|u_{0}\right\|_{s}\right) e^{-\lambda^{\prime \prime}\left(t-t_{0}\right)}\left\|u_{0}\right\|_{s} \quad \text { for all } t \geq t_{0}
$$

where $\gamma_{s}$ is a nondecreasing continuous function.

Proof. First, proceeding as for Theorem 4.1, we check that the system (5.10) is globally well-posed in $H_{0}^{s}(\mathbb{T})$. Next, rough estimates for $\|u(., t)\|_{s}$ are established for the times $t$ when both $K_{\lambda}$ and $G G^{*}$ are active.

Lemma 5.2. Pick any pair $\left(t_{0}, u_{0}\right) \in \mathbb{R} \times H_{0}^{s}(\mathbb{T})$. Then the system (5.10) admits a unique solution $u: \mathbb{T} \times\left[t_{0},+\infty\right) \rightarrow \mathbb{R}$ fulfilling

$$
u \in Z_{\frac{1}{2}, s}^{\left[t_{0}, t_{0}+T\right]} \cap L^{2}\left(t_{0}, t_{0}+T ; L_{0}^{2}(\mathbb{T})\right) \quad \text { for all } T>0 .
$$

The following a priori estimates hold true

$$
\begin{array}{ll}
\text { If } \quad\left\|u_{0}\right\|_{s} \leq 1, \quad\|u(., t)\|_{s} \leq \alpha_{s}(1) \quad \text { for all } t \geq t_{0} ; \\
\text { If } \quad\left\|u_{0}\right\|_{s}>1, \quad\|u(., t)\|_{s} \leq \alpha_{s}\left(\left\|u_{0}\right\| \|_{0}\right)\left\|u_{0}\right\|_{s} \quad \text { for all } t \geq t_{0} ; \\
\text { If } \quad\left\|u_{0}\right\|_{s} \leq R, \quad\|u(., t)\|_{s} \leq K_{s} e^{d_{s}\left(t-t_{0}\right)}\left\|u_{0}\right\|_{s} \quad \text { for all } t \geq t_{0},
\end{array}
$$

where $K_{s}$ and $d_{s}$ denote some positive constants depending only on $s$ and $R$. 
Proof of Lemma 5.2: Let us begin with the local existence of a solution. Pick any pair $\left(t_{0}, u_{0}\right) \in$ $\mathbb{R} \times H_{0}^{s}(\mathbb{T})$. It may be seen that

$$
\left\|K\left(v_{1}, t\right)-K\left(v_{2}, t\right)\right\|_{s} \leq c\left\|v_{1}-v_{2}\right\|_{s} \quad \text { for all } v_{1}, v_{2} \in H_{0}^{s}(\mathbb{T}), t \in \mathbb{R}
$$

where $c$ denotes a positive constant independent of $v_{1}, v_{2}$ and $t$. Defining the map

$$
\Gamma(v)(t)=W\left(t-t_{0}\right) u_{0}-\int_{t_{0}}^{t} W(t-\tau)\left(v \partial_{x} v\right)(\tau) d \tau-\int_{t_{0}}^{t} W(t-\tau) K(v(\tau), \tau) d \tau
$$

we infer as in the proof of Theorem 4.1 that (4.6) and (4.7) hold for all $v, v_{1}, v_{2} \in Z_{\frac{1}{2}, s}^{\left[t_{0}, t_{0}+\tilde{T}\right]} \cap$ $L^{2}\left(t_{0}, t_{0}+\tilde{T} ; L_{0}^{2}(\mathbb{T})\right)$. Moreover, the involved constants only depend on $\theta$ for its $L^{\infty}$ norm and not on $\delta$. Let $d=2 C_{1}\left\|u_{0}\right\|_{s}$ and $\tilde{T}>0$ be such that

$$
2 C_{2} d \tilde{T}^{\theta}+C_{3} \tilde{T}^{1-\varepsilon} \leq \frac{1}{2}
$$

Then the map $\Gamma$ is a contraction in the closed ball $B_{d}(0)$ of $Z_{\frac{1}{2}, s}^{\left[t_{0}, t_{0}+\tilde{T}\right]} \cap L^{2}\left(t_{0}, t_{0}+\tilde{T} ; L_{0}^{2}(\mathbb{T})\right)$ for the $\|v\|_{Z_{\frac{1}{2}, s}^{\left[t_{0}, t_{0}+\tilde{T}\right]}}$ norm. Its fixed point is the desired solution of (5.10). Note that for some constant $C_{4}>0$ we have that

$$
\|u\|_{L^{\infty}\left(t_{0}, t_{0}+\tilde{T} ; H^{s}(\mathbb{T})\right)} \leq C_{4}\|u\|_{Z_{\frac{1}{2}, s}^{\left[t_{0}, t_{0}+\tilde{T}\right]}} \leq 2 C_{1} C_{4}\left\|u_{0}\right\|_{s}
$$

Noticing that $K(u, t)=G G^{*} u$ for $\|u\|_{s}>1$ and using (5.2), we infer that the solution $u$ of (5.10) is defined for all $t \geq t_{0}$. Moreover, (5.2) yields (5.12) and (5.13). Let

$$
d^{\prime} 2 C_{1} \max \left(\alpha_{s}(1), \alpha_{s}\left(\left\|u_{0}\right\|_{0}\right)\left\|u_{0}\right\|_{s}\right) .
$$

Note that $d^{\prime}$ depends only on $R$ and $s$. Replacing $\tilde{T}$ by $T^{\prime}$ satisfying

$$
2 C_{2} d^{\prime} T^{\prime \theta}+C_{3} T^{1-\varepsilon} \leq \frac{1}{2}
$$

in the application of the contraction mapping principle, we infer that the (unique) solution $u$ of (5.10) fulfills

$$
\|u\|_{Z_{\frac{1}{2}, s}^{\left[t_{0}+k T^{\prime}, t_{0}+(k+1) T^{\prime}\right]}} \leq 2 C_{1}\left\|u\left(., t_{0}+k T^{\prime}\right)\right\|_{s} .
$$

This gives

$$
\|u\|_{L^{\infty}\left(t_{0}+k T^{\prime}, t_{0}+(k+1) T^{\prime}, H^{s}(\mathbb{T})\right)} \leq 2 C_{1} C_{4}\left\|u\left(., t_{0}+k T^{\prime}\right)\right\|_{s}
$$

and

$$
\|u(., t)\|_{s} \leq K_{s} e^{d_{s}\left(t-t_{0}\right)}\left\|u_{0}\right\|_{s}
$$

for some constants $K_{s}>0, d_{s}>0$ depending only on $s$ and $R$.

Given $\lambda^{\prime \prime}$ as in the statement of the theorem, we pick $\delta>0$ such that

$$
\lambda^{\prime \prime}<-2 \delta d_{s}+(1-2 \delta) \frac{\kappa+\lambda^{\prime}}{2} \quad \text { and } \quad \delta d_{s}-(1-2 \delta) \kappa<0 .
$$

Next, choose $r_{1} \in\left(0, r_{0}\right)$ such that

$$
\alpha_{s}\left(\alpha_{s}(1)\right) C_{s} K_{s}^{4} e^{4 \delta T d_{s}} r_{1}<r_{0}
$$


and $T_{0}>0$ such that

$$
\begin{aligned}
\alpha_{s}(1) \alpha_{s}\left(\alpha_{s}(1)\right) K_{s} e^{\left[\delta d_{s}-(1-2 \delta) \kappa\right] T} & \leq r_{1}, \\
\alpha_{s}(1) C_{s} K_{s}^{4} e^{\left[4 \delta d_{s}-(1-2 \delta)\left(\kappa+\lambda^{\prime}\right)\right] T} & \leq e^{-2 \lambda^{\prime \prime} T}
\end{aligned}
$$

for all $T \geq T_{0}$. Note that $T_{0}$ exists by (5.15). Pick any $u_{0} \in H_{0}^{s}(\mathbb{T})$ and any time $t_{0} \in \mathbb{R}$. The proof rests on a series of claims.

Claim 1. There exists a time $t_{1} \in\left[t_{0}, t_{0}+\kappa^{-1} \ln \left(\alpha_{s}\left(\left\|u_{0}\right\|_{0}\right)\left\|u_{0}\right\|_{s}\right)\right]$ such that

$$
\left\|u\left(t_{1}\right)\right\|_{s} \leq 1 \text {. }
$$

Without loss of generality we may assume that $\left\|u\left(t_{0}\right)\right\|_{s} \geq 1$. Then the dynamics of $u$ is governed by (5.1) as long as $\|u(t)\|_{s} \geq 1$. By (5.2), we have (5.19) for some time $t_{1}$ with

$$
\alpha_{s}\left(\left\|u_{0}\right\|_{0}\right) e^{-\kappa\left(t_{1}-t_{0}\right)}\left\|u_{0}\right\|_{s} \leq 1 .
$$

Therefore, Claim 1 holds.

Claim 2. There exists a time $t_{2} \in 2 \mathbb{Z T} \cap\left[t_{1}, t_{1}+3 T\right]$ such that

$$
\left\|u\left(t_{2}\right)\right\|_{s} \leq r_{1} .
$$

From the fact that $\left\|u\left(t_{1}\right)\right\|_{0} \leq 1$ and (5.2) we have that

$$
\|u(t)\|_{s} \leq \alpha_{s}(1) \quad \text { for all } t \geq t_{1} .
$$

Pick $R=\alpha_{s}(1)$ and let $K_{s}$ and $d_{s}$ be as given in Lemma 5.2 for that choice of $R$. Let $t_{1}^{\prime} \geq t_{1}$ denote the first time of the form $t_{1}^{\prime}=(2 k+1) T+\delta$ with $k \in \mathbb{Z}$, and let $t_{2}=(2 k+2) T$. Then it follows from (5.2), (5.14) and (5.17) that

$$
\left\|u\left(t_{2}\right)\right\|_{s} \leq K_{s} e^{\delta T d_{s}} \alpha_{s}\left(\alpha_{s}(1)\right) e^{-\kappa(1-2 \delta) T}\left\|u\left(t_{1}^{\prime}\right)\right\|_{s} \leq r_{1} .
$$

ClAim 3. $\|u(t)\|_{s} \leq r_{0}$ for all $t \geq t_{2}$ and $\left\|u\left(t_{2}+2 k T\right)\right\|_{s} \leq e^{-2 k \lambda^{\prime \prime} T}\left\|u\left(t_{2}\right)\right\|_{s}$ for all $k \in \mathbb{N}$.

First, we notice that the dynamics of $u$ is governed by (5.3) (resp. by (5.1)) when $t \in\left(t_{2}+\right.$ $\left.\delta T, t_{2}+(1-\delta) T\right)\left(\right.$ resp. when $\left.t \in\left(t_{2}+(1+\delta) T, t_{2}+(2-\delta) T\right)\right)$, as long as $\|u(t)\|_{s} \leq r_{0}$. Therefore, using (5.2), (5.4), (5.14), and (5.16) we obtain that

$$
\|u(t)\|_{s} \leq\left(\alpha_{s}\left(\alpha_{s}(1)\right) K_{s}^{2} e^{2 \delta T d_{s}}\right)\left(C_{s} K_{s}^{2} e^{2 \delta T d_{s}}\right)\left\|u\left(t_{2}\right)\right\|_{s}<r_{0} \quad \text { for all } t \in\left[t_{2}, t_{2}+2 T\right] .
$$

On the other hand, by (5.18),

$$
\begin{aligned}
\left\|u\left(t_{2}+2 T\right)\right\|_{s} & \leq\left(\alpha_{s}(1) e^{-\kappa(1-2 \delta) T} K_{s}^{2} e^{2 \delta T d_{s}}\right)\left(C_{s} e^{-\lambda^{\prime}(1-2 \delta) T} K_{s}^{2} e^{2 \delta T d_{s}}\right)\left\|u\left(t_{2}\right)\right\|_{s} \\
& \leq e^{-2 \lambda^{\prime \prime} T}\left\|u\left(t_{2}\right)\right\|_{s} \\
& \leq r_{1} .
\end{aligned}
$$

The claim follows by an obvious induction.

It follows from Claim 3 that for $t \geq t_{2}$

$$
\|u(t)\|_{s} \leq c e^{-\lambda^{\prime \prime}\left(t-t_{2}\right)}\left\|u\left(t_{2}\right)\right\|_{s}
$$

for some constant $c$ independent of $t$ and $u_{0}$. Since

$$
t_{2}-t_{0} \leq 3 T+\kappa^{-1} \ln \left(\alpha_{s}\left(\left\|u_{0}\right\|_{0}\right)\left\|u_{0}\right\|_{s}\right),
$$

the theorem follows. 


\section{Remark 5.3.}

- A natural idea to combine both feedback controls would be to consider a discontinuous feedback control which agrees with $K_{0} u$ when $\|u\|_{s}$ is large, and with $K_{\lambda} u$ when $\|u\|_{s}$ is small. The main difficulty is then to define properly what we mean by a solution of the closed-loop system. In finite dimension, the Filippov solutions are widely used by the control community to deal with discontinuous systems. (See [9] for the definition of a Filippov solution.) The main advantage of the time-varying feedback law considered here is its regularity, which guarantees the existence and uniqueness of "classical" solutions for the closed-loop system.

- It would be interesting to see whether a smooth time-invariant feedback law ensuring a semiglobal exponential stabilization with an arbitrary decay rate can be designed.

- A simpler, but less efficient, time-varying feedback law is

$$
K(u, t):=\theta\left(\frac{t}{T}\right) \rho\left(\|u\|_{s}^{2}\right) K_{\lambda} u+\theta\left(\frac{t-T}{T}\right) G G^{*} u .
$$

Acknowledgments: This work is partially conducted while the third author (BZ) was visiting Laboratoire Jacques-Louis Lions of Université Pierre et Marie Curie in May and June of 2008, and the Yantz Center of Mathematics of Sichuan University in November of 2008. He thanks both institutions for their hospitality. BZ was partially supported by the Paris Foundation of Mathematics, the Charles Phelps Taft Memorial Fund at the University of Cincinnati and the Chunhui program (State Education Ministry of China) under grant Z007-1-61006

\section{References}

[1] Bergh, J. and Löfstrom, J., Interpolation Spaces, An Introduction, Springer Verlag 1976

[2] Bona, J. L., Sun, S. M. and Zhang, B.-Y., The initial-boundary value problem for the Kortewegde Vries equation in a quarter plane, Trans. American Math. Soc. 354(2001), 427-490.

[3] Bourgain, J., Fourier transform restriction phenomena for certain lattice subsets and applications to non-linear evolution equations, part II: the KdV equation, Geom. E Funct. Anal. 3 (1993), $209-262$.

[4] Boussinesq, J., Essai sur la théorie des eaux courantes; Mémoires présentés par divers savants à l'Acad. des Sci. Inst. Nat. France, 23 (1877), 1 C680.

[5] Cerpa, E., and Crépeau, E., Boundary controllability for the nonlinear Korteweg-de Vries equation on any critical domain, Ann. I.H. Poincaré - AN (2008), doi:10.1016/j.anihpc.2007.11.003.

[6] Cerpa, E., and Crépeau, E., Rapid exponential stabilization for a linear Korteweg-de Vries equation, Discrete Contin. Dyn. Syst. Ser. B, 11 (2009), no. 3, 655-668.

[7] Colliander J., Keel M., Staffilani G., Takaoka H., and Tao T., Sharp global well-posedness for $K d V$ and modified $K d V$ on $\mathbb{R}$ and $\mathbb{T}$, Journal of the AMS 16 (2003), No. 3, 705-749.

[8] Coron, J.-M. and Crépeau, E., Exact boundary controllability of a nonlinear KdV equation with a critical length, J. Eur. Math. Soc., 6 (2004), 367-398. 
[9] Coron, J.-M. and Rosier, L., A Relation Between Continuous Time-Varying and Discontinuous Feedback Stabilization, Journal of Mathematical Systems, Estimation, and Control, 4(1994), $67-84$.

[10] Dehman, B., Gérard, P., Lebeau, G., Stabilization and control for the nonlinear Schrödinger equation on a compact surface, Math. Z 254(2006), 729-749.

[11] B. Dehman, G. Lebeau and E. Zuazua : Stabilization and control for the subcritical semilinear wave equation. Anna. Sci. Ec. Norm. Super. 36:525-551 (2003).

[12] Gardner, C. S., Greene, J. M., Kruskal, M. D. and Miura, R. M., Method for solving the Korteweg-de Vries equation, Phys. Rev. Lett., 19 (1967), 1095-1097.

[13] Glass, O., and Guerrero, S., Some exact controllability results for the linear KdV equation and uniform controllability in the zero-dispersion limit, Asymptot. Anal. 60 (2008), no. 1-2, 61-100.

[14] de Jager, E. M., On the origin of the Korteweg-de Vries equation, arXiv:math.HO/0602661

[15] Kappeler, T. and Topalov, P., Well-posedness of $K d V$ on $H^{-1}(\mathbb{T})$, Duke Math. J., 135 (2006), 327-360.

[16] Kato, T., On the Cauchy problem for the (generalized) Korteweg-de Vries equations, in Advances in Mathematics Supplementary Studies, Stud. Appl. Math. 8, Academic Press, New York, 1983, 93-128.

[17] Kenig, C. E., Ponce, G. and Vega, L., Well-posedness of the initial value problem for the KdV equation, J. Amer. Math. Soc., 4 (1991), 323-347.

[18] Kenig, C. E., Ponce, G. and Vega, L., A bilinear estimate with applications to the KdV equation, J. Amer. Math. Soc., 9 (1996), 573-603.

[19] Korteweg, D. J. and de Vries, G., On the change of form of long waves advancing in a rectangular canal, and on a new type of long stationary waves, Philos. Mag., 39 (1895), 422-443.

[20] Laurent, C., Global controllability and stabilization for the nonlinear Schrödinger equation on an interval, ESAIM Control Optim. Cal. Var., in press.

[21] Laurent, C., Global controllability and stabilization for the nonlinear Schrödinger equation on some compact manifolds of dimension 3, submitted.

[22] Linares, F., and Pazoto, A. F., On the exponential decay of the critical generalized Korteweg-de Vries equation with localized damping, Proc. Amer. Math. Soc. 135 (2007), no. 5, 1515-1522.

[23] Linares, F., and Pazoto, A. F., Asymptotic behavior of the Korteweg-de Vries equation posed in a quarter plane, J. Diff. Equations 246 (2009) 13421353.

[24] Micu, S., Ortega, J., Rosier, L., and Zhang, B.-Y., Control and stabilization of a family of Boussinesq systems, Discrete and Continuous Dynamical Systems, 24 (2009), no. 2, 273-313.

[25] Miura, R. M., The Korteweg-de Vries equation: A survey of results, SIAM Rev., 18 (1976), 412-459.

[26] Pazoto, A. F., Unique continuation and decay for the Korteweg-de Vries equation with localized damping, ESAIM Control Optim. Calc. Var., 11 (2005), pp. 473-486. 
[27] Pazoto, A. F. and Rosier, L., Stabilization of a Boussinesq system of KdV-KdV type, Systems \& Control Letters 57 (2008), 595-601.

[28] Perla-Menzala, G., Vasconcellos, C. F., and Zuazua, E., Stabilization of the Korteweg-de Vries equation with localized damping, Quart. Appl. Math., 60 (2002), pp. 111-129.

[29] Russell, D. L, D. L. Computational study of the Korteweg-de Vries equation with localized control action, Distributed Parameter Control Systems: New Trends and Applications, G. Chen, E. B. Lee, W. Littman, and L. Markus, eds., Lecture Notes in Pure and Appl. Math., vol. 128, Marcel Dekker, New York, 1991, 195-203.

[30] Rosier, L., Exact boundary controllability for the Korteweg-de Vries equation on a bounded domain, ESAIM Control Optim. Cal. Var., 2 (1997), 33-55.

[31] Rosier, L., Exact boundary controllability for the linear Korteweg-de Vries equation on the half-line, SIAM J. Control Optim. 39 (2000) 331-351.

[32] Rosier, L., Control of the surface of a fluid by a wavemaker, ESAIM Control Optim. Cal. Var. 10 (2004), 346-380.

[33] Rosier, L. and Zhang, B.-Y., Global stabilization of the generalized Korteweg-de Vries equation, SIAM J. Control Optim. 45 (2006), no. 3, 927-956.

[34] Rosier, L. and Zhang, B.-Y., Exact controllability and stabilization of the nonlinear Schrödinger equation on a bounded interval, SIAM J. Control Optim. 48 (2009), no. 2, 972-992.

[35] Rosier, L. and Zhang, B.-Y., Exact boundary controllability of the nonlinear Schrödinger equation, J. Differential Equations 246 (2009), 4129-4153.

[36] Rosier, L. and Zhang, B.-Y., Control and stabilization of the nonlinear Schrödinger equation on rectangles, submitted.

[37] Russell, D. L. and Zhang, B.-Y., Controllability and stabilizability of the third order linear dispersion equation on a periodic domain, SIAM J. Cont. Optim., 31 (1993), 659-676.

[38] Russell, D. L. and Zhang, B.-Y., Exact controllability and stabilizability of the Korteweg-de Vries equation, Trans. Amer. Math. Soc., 348 (1996), 3643-3672.

[39] Saut, J.-C. and Temam, R., Remarks on the Korteweg-de Vries equation, Israel J. Math., 24 (1976), 78-87.

[40] Saut, J.-C. and Scheurer, B., Unique continuation for some evolution equations, J. Differential Equations, 66 (1987), no. 1, 118-139.

[41] Slemrod, M., A note on complete controllability and stabilizability for linear control systems in Hilbert space, SIAM Control, 12 (1974), 500-508.

[42] Tao, T., Nonlinear dispersive equations, Local and global analysis. CBMS Regional Conference Series in Mathematics, 106. AMS, Providence, RI, 2006.

[43] Tartar, L., Interpolation non linéaire et régularité, J. Funct. Analys, 9(1972), 469-489.

[44] Temam, R., Sur un problème non linéaire, J. Math. Pures Appl., 48(1969), 159-172. 
[45] Zhang, B.-Y., Unique continuation for the Korteweg-De Vries equation, SIAM J. Math. Anal., 23 (1992), 55-71.

[46] Zhang, B.-Y., Taylor series expansion for solutions of the Korteweg-de Vries equation with respect to their initial values, J. Funct. Anal., 129 (1995), 293-324.

[47] Zhang, B.-Y., Exact boundary controllability of the Korteweg-de Vries equation, SIAM J. Cont. Optim., 37 (1999), 543-565. 